\title{
Philosophiques
}

\section{Délibération démocratique et ontologie sociale}

\section{David Kahane}

Volume 29, numéro 2, automne 2002

\section{La démocratie délibérative}

URI : https://id.erudit.org/iderudit/006254ar

DOI : https://doi.org/10.7202/006254ar

Aller au sommaire du numéro

\section{Éditeur(s)}

Société de philosophie du Québec

\section{ISSN}

0316-2923 (imprimé)

1492-1391 (numérique)

Découvrir la revue

\section{Citer cet article}

Kahane, D. (2002). Délibération démocratique et ontologie sociale. Philosophiques, 29(2), 251-286. https://doi.org/10.7202/006254ar

\section{Résumé de l'article}

Les théoriciens de la démocratie s'appuient, implicitement ou explicitement, sur des ontologies sociales, c'est-à-dire sur une conception de la relation constitutive entre individus et groupes sociaux. Cet article met en évidence l'ontologie sociale « individualiste " implicite dans les sondages d'opinion délibératifs de James Fishkin pour ensuite proposer une alternative : l'ontologie sociale " relationnelle ». L'article examine la façon dont ce second type d'ontologie sociale modifierait les principes et les mécanismes délibératifs, de même que les coûts en légitimité qu'impliquerait la mise en question de l'individualisme normatif. En particulier, le passage d'une ontologie sociale individualiste à une ontologie sociale relationnelle place au centre des théories de la démocratie des analyses controversées du pouvoir et de l'oppression. Je suggère que cette politisation est nécessaire pour que les approches délibératives soient adéquates tant au type d'êtres que nous sommes qu'aux rapports de force sociaux dans lesquels nous nous mouvons. Sans cette politisation, la démocratie délibérative ne pourrait pas davantage répondre aux exigences de la justice démocratique. 


\title{
Délibération démocratique et ontologie sociale
}

\author{
DAVID KAHANE \\ University of Alberta \\ david.kahane@ ualberta.ca
}

\begin{abstract}
RÉSUMÉ. - Les théoriciens de la démocratie s'appuient, implicitement ou explicitement, sur des ontologies sociales, c'est-à-dire sur une conception de la relation constitutive entre individus et groupes sociaux. Cet article met en évidence l'ontologie sociale « individualiste» implicite dans les sondages d'opinion délibératifs de James Fishkin pour ensuite proposer une alternative : I'ontologie sociale « relationnelle». L'article examine la façon dont ce second type d'ontologie sociale modifierait les principes et les mécanismes délibératifs, de même que les coûts en légitimité qu'impliquerait la mise en question de l'individualisme normatif. En particulier, le passage d'une ontologie sociale individualiste à une ontologie sociale relationnelle place au centre des théories de la démocratie des analyses controversées du pouvoir et de l'oppression. Je suggère que cette politisation est nécessaire pour que les approches délibératives soient adéquates tant au type d'êtres que nous sommes qu'aux rapports de force sociaux dans lesquels nous nous mouvons. Sans cette politisation, la démocratie délibérative ne pourrait pas davantage répondre aux exigences de la justice démocratique.
\end{abstract}

\begin{abstract}
Democratic theorists rely (implicitly or explicitly) on social ontologies, i.e., accounts of the constitutive relationship between individuals and social groups. This article draws out the "individualist" social ontology implicit in James Fishkin's "deliberative opinion polls", then argues for a rival, "relational" social ontology. The article explores how a relational social ontology would change deliberative principles and mechanisms, as well as the legitimacy costs of this move away from normative individualism. In particular, the move from an individualist to a relational social ontology brings controversial analyses of power and oppression to the center of deliberative democratic theories; I suggest that this politicization is necessary if deliberative approaches are to be adequate to the kinds of beings we are, the power-laden social relations within which we move, and the demands of democratic justice.
\end{abstract}

Le tournant en faveur de la délibération qu'a connu la philosophie politique récente a ses racines tant dans la théorie que la pratique. Les théoriciens ont reconnu qu'il fallait renoncer à l'espoir des libéraux de justifier rationnellement des normes de justice acceptables pour tous les citoyens. Le pluralisme moral et social atteint à une telle profondeur qu'il ne semble pas y avoir de conception unique de la raison sur laquelle fonder le projet de justification libéral. II n'apparaît pas clairement non plus qu'une conception de la raison puisse engendrer des principes de justice sans s'appuyer - implicitement ou explicitement - sur des valeurs relevant d'un cadre culturel ou historique spécifique. En pratique, on reconnaît que les démocraties nord-américaines sont marquées par de grossiers déficits au plan de la délibération et par conséquent on mesure les coûts dont les sociétés multiculturelles font les frais en termes de légitimité et de cohésion sociale. 
Je fais miennes ces analyses théoriques et pratiques sur les lacunes des approches non délibératives et partage l'opinion favorable à la délibération démocratique. $D$ ans cet article, cependant, je veux faire valoir que dans la mesure où nous nous tournons vers la délibération, il est important que nous le fassions munis d'une conception adéquate des groupes sociaux. Plus spécifiquement, je veux suggérer que sans une ontologie sociale qui aborde convenablement les groupes et soit sensible aux rapports de pouvoirs, tant notre travail théorique que nos recommandations pratiques sur la délibération ont de bonnes chances d'être faussés. J'entends formuler les liens entre normes de délibération et ontologie sociale à travers l'examen d'un modèle de délibération qui a fait date, celui de James Fishkin.

Dans les pages qui vont suivre, j'offre un aperçu général de la description que fait Fishkin des normes sociales et des «sondages d'opinion délibératifs» qu'il propose en vue de véhiculer ces normes. Je distingue ensuite trois grandes approches en ontologie sociale, chacune décrivant de manière différente les rapports constitutifs entre individus et groupes sociaux. Je ferai valoir que les sondages délibératifs s'appuient implicitement sur une ontologie sociale individualiste et, par le fait même, se font une conception erronée de la pertinence des groupes sociaux et du pouvoir social dans la constitution des institutions délibératives. J'explorerai la façon dont les sondages délibératifs pourraient être remodelés à la lumière d'une ontologie sociale relationnelle, afin de mieux combler les fossés épistémiques et surmonter les asymétries en termes de pouvoirs caractérisant les sociétés culturellement diversifiées.

\section{Fishkin sur les normes et les processus démocratiques}

Dans The D ialogue of J ustice ${ }^{1}$, James Fishkin pose les bases normatives et méthodologiques de sa théorie de la démocratie délibérative. D'une part, il rejette le «libéralisme systématique», lequel décrit l'idéal politique en termes de principes premiers ne souffrant pas d'exceptions, offre des critères de progrès moral et traite de ses conseils au plan normatif comme s'ils étaient indépendants du contexte. D'autre part, Fishkin plaide plutôt en faveur d'un «libéralisme limité» mettant l'accent sur les procédures démocratiques légitimement appelées à arbitrer les conflits de valeurs dans des contextes particuliers. II met l'accent sur les normes auxquelles obéit une bonne délibération politique et sur les réformes institutionnelles susceptibles d'y conduire. ${ }^{2}$

1. James Fishkin (1992), The D ialogue of J ustice. Toward a Self-R eflective Society, N ew $\mathrm{H}$ aven, Yale University Press.

Toutes les traductions en français sont de nous. (N.d.T.)

2. En mettant ainsi l'accent sur le processus démocratique aux dépens des principes devant guider son issue, Fishkin s'accorde avec une large mouvance en théorie politique contemporaine, laquelle englobe la théorie démocratique délibérative de même que des versions pluralistes et contextualistes du radicalisme politique. Je me situe dans cette même mouvance. Le travail de Fishkin, cependant, est particulièrement instructif pour plusieurs raisons : d'abord, il 
Il décrit trois conditions de la démocratie, soit les « exigences qui doivent être remplies pour que la voix du peuple mérite d'être entendue ${ }^{3}$ : l'égalité, la non-tyrannie et la délibération. Chacune de ces conditions vise à assurer que les divers intérêts et perspectives de la population influencent comme il convient la prise de décisions. La norme d'égalité comprend : les règles formelles de décision, accordant un poids égal aux préférences de chaque citoyen ; les conditions d'isolement, visant à empêcher que les inégalités sociales et économiques n'entachent les processus démocratiques; les garanties assurant que chacun puisse être effectivement entendu de manière que « toute la gamme des intérêts ayant un nombre significatif de partisans [... ] soient suffisamment entendus pour que les gens aient l'occasion de trancher entre eux ${ }^{4}$. La seconde condition, la non-tyrannie, assure qu'aucune politique n'impose de graves privations à un groupe donné qu'une autre politique lui aurait épargnées. Ici, Fishkin vise essentiellement à se faire le champion des droits fondamentaux. La troisième condition à la démocratie est la délibération, mécanisme permettant d'élever et purifier I'opinion. Fishkin cite Robert Dahl : «Afin d'exprimer de manière précise leurs préférences, tous les citoyens et citoyennes doivent disposer d'occasions égales et adéquates pour découvrir et confirmer quelles sont leurs préférences concernant le point à trancher, dans le temps limité qu'implique la prise de décision ${ }^{5}$.

Selon Fishkin, la politique américaine contemporaine s'est éloignée de la délibération, les discussions et décisions publiques reflétant de plus en plus les

tire systématiquement les conséquences pratiques de ses engagements en matière de démocratie délibérative; ensuite, son travail théorique et pratique illustre de bout en bout une conception individualiste des agents politiques, ce qui nous permet de mieux voir en quoi les descriptions de la délibération démocratique sont façonnées par leur ontologie sociale sous-jacente.

Sur la théorie démocratique délibérative, voir par exemple : Bohman, James, Public D eliberation. Pluralism, Complexity, and D emocracy, Cambridge, M ass., M IT Press, 1996; Bohman, James et Rehg, William, dir., D eliberative D emocracy. Essays on Reason and Politics, Cambridge, M ass., M IT Press, 1997 ; Chambers, Simone, Reasonable D emocracy. Jürgen H abermas and the Politics of D iscourse, Ithaca, Cornell University Press, 1996; Elster, Jon, dir., D eliberative D emocracy, Cambridge, Cambridge University Press, 1998; H abermas, Jürgen, «Discourse Ethics. N otes on a Program of Philosophical Justification », dans N icholsen, S., dir., M oral Consciousness and Communicative Action, Cambridge, M ass., M IT Press, 1990; Valadez, Jorge M ., D eliberative D emocracy, Political L egitimacy, and Self-D etermination in M ulticultural Societies, Boulder, Westview, 2001. Sur la théorie pluraliste, voir par exemple : Tully, James, Strange M ultiplicity. Constitutionalism in an A ge of D iversity, Cambridge, Cambridge University Press, 1990; Williams, M elissa M., Voice, Trust, and M emory. M arginalized G roups and the Failings of Liberal Representation, Princeton, Princeton University Press, 1998; Young, Iris M arion, Inclusion and D emocracy, O xford, Oxford University Press, 2000.

3. Fishkin, D emocracy and D eliberation. N ew Directions for D emocratic R eforms, $\mathrm{N}$ ew H aven, Yale University Press, 1991, p. 104.

4. Ibid., p. 33, 31. Dans The Voice of People, Fishkin distingue un quatrième critère dit de participation, qui exige qu' 'une proportion significative des citoyens s'impliquent dans le processus» pour des raisons de légitimation. Cf. Fishkin, The Voice of People. Public O pinion and Democracy, N ew H aven, Yale University Press, 1995, p. 34.

5. Fishkin, 1991, p. 36. 
opinions irréfléchies de citoyens ne connaissant rien aux points débattus ${ }^{6}$. Les représentants démocratiquement élus tendent à se laisser mener par les sondages, et cette tendance plébiscitaire se combine à des institutions gouvernées par le principe majoritaire pour «vider le système de toute délibération effective en apportant le pouvoir aux masses dans des conditions telles que les masses ont peu de chances de délibérer sur le pouvoir qu'elles exercent ${ }^{7}$.

II se fait ici l'écho des propos d'A mitai Etzioni voulant que sans délibération, les positions des citoyens ont de bonnes chances d'être « impulsives, mal informées et inutilement polarisées ${ }^{8}$. Fishkin est largement tributaire d'études empiriques de l'opinion politique qui (bien que contestées) montrent combien «les citoyens des publics de masse ne se distinguent pas précisément par leurs connaissances, leur sophistication ou la cohérence de leurs croyances et opinions. Sur bien des questions, quatre citoyens sur cinq n'ont pas d'opinions stables, non aléatoires; ils ont ce que les psychologues politiques appellent des "non-attitudes" ou "pseudo-opinions" »9. Une telle ignorance politique peut être rationnelle pour l'individu, la structure de la politique électorale étant ce qu'elle est : le vote de chacun des citoyens a si peu de poids que cela constitue un bien faible incitatif à cultiver sa connaissance des événements politiques.

De l'avis de Fishkin : "C'est une réalisation douteuse que de donner du pouvoir aux gens dans des conditions telles qu'ils ne sont pas vraiment en mesure de songer à la façon dont ils devraient I'exercer ${ }^{10}$. Sa vision globale suggère que lors d'une bonne prise de décision démocratique, l'issue des discussions reflétera la prépondérance de l'opinion dans une société où les opinions sont informées et ne désavantagent pas indûment les minorités : "Le public ne peut jamais mieux parler en son nom que lorsqu'il peut se réunir de quelque manière pour entendre les arguments sur les diverses facettes d'un problème et, au terme d'une discussion face à face, en arriver à une décision collective» ${ }^{11}$.

6. Pour une description en termes forts de cette tendance, voir Ackerman, Bruce et Fishkin, James, «D eliberation Day», conférence sur D eliberating about D eliberative D emocracy, 2000, http ://www.la.utexas.edu/conf2000/papers/D eliberationDay.pdf. Dans The Voice of People, Fishkin impute le passage de la délibération au sein de l'élite à la déférence vis-à-vis de l'opinion de la masse à des glissements dans des institutions représentatives clés telles le collège électoral.

7. Fishkin, 1991, p. 20.

8. I bid., p. 98.

9. I bid., p. 83. Voir aussi Zaller, John, R., The $\mathrm{N}$ ature and $\mathrm{O}$ rigins of $\mathrm{M}$ ass $\mathrm{O}$ pinion, Cambridge, Cambridge University Press, 1992. Contre ce point de vue, voir Gardner, Elizabeth et Lupia, Arthur, « Voter Competence in Direct Legislative Elections», dans Elkin, S. L. et Soltan, K. E., dir., Citizen Competence and D emocratic Institutions, University Park, Penn State University Press, 1999, p. 147-160; N ew port, Frank, «W hy do we need a Deliberative Poll?», The Public Perspective, janvier 1996, p. 7-9; Page, Benjamin et Dimock, M ichael, «Political Knowledge and Citizen Competence», dans Elkin et Soltan, dir., 1999, p. 117-146; Tringali, Brian C., «Experimenting with Artificial Democracy », The Public Perspective, janvier 1996, p. 19-20.

10. Fishkin, 1991, p. 21.

11. Fishkin, 1995, p. 4. 
Un sondage d'opinion délibératif rassemble une coupe transversale significative de la communauté politique pour délibérer sur une grande question politique. Fishkin a contribué à organiser de tels sondages en $\mathrm{G}$ rande-Bretagne afin de discuter du système de justice pénale (1994), des relations de la nation avec l'Europe (1995), de l'avenir de la monarchie (1996), de questions d'économie politique (1997) et du rationnement des services offerts par le système public d'assurance-santé (1998); il a fait de même en Australie afin de déterminer si le pays devait devenir une république (1999) et aux États-Unis sur des questions relatives à la réglementation de services comme l'électricité et le gaz au Texas (1996-1998). Dans chacun de ces sondages délibératifs, un échantillon statistiquement représentatif de la communauté pertinente a été sélectionné et sondé sur les questions à l'étude. Les sujets ont été réunis pour deux à trois jours d'intense délibération puis sondés à nouveau afin de relever les différences entre les opinions irréfléchies qu'ils soutenaient d'entrée de jeu et celles filtrées par la délibération au terme du processus. Comme l'écrivent Ackerman et Fishkin :

Les participants sont effectivement motivés à prêter attention, ils ont accès à de bonnes informations, discutent des problèmes avec les autres, posent des questions aux experts ou aux preneurs de décision de tous bords et en viennent à poser un jugement réfléchi. Bref, à quoi ressemblerait l'opinion politique si les répondants étaient motivés à surmonter les incitatifs à l'ignorance rationnelle pour se comporter un peu plus comme des citoyens idéaux ? ${ }^{12}$

Q u'il me soit permis de décrire plus en détail l'exercice. A près quoi, je montrerai en quoi il s'appuie sur une ontologie sociale problématique.

\section{La sélection des participants}

Un sondage d'opinion délibératif commence par la sélection d'une coupe transversale statistiquement représentative de l'électorat approprié (par exemple, dans les cas britanniques et australien, la nation entière; dans l'exemple texan, les citoyens de l'État); les organisateurs « se servent des techniques d'échantillonnage mises au point par la recherche sur l'opinion publique pour représenter et réunir une version de ce que nous sommes tous» ${ }^{13}$. À travers des entrevues à la maison et des enquêtes écrites, les participants, sélectionnés de manière aléatoire, sont interrogés en détail sur leurs connaissances et leur attitude concernant les problèmes politiques en question. Ce premier échantillonnage établit un point de référence sur l'opinion publique non infléchie par une délibération hors de l'ordinaire.

En attendant la délibération

Les répondants sont ensuite invités à prendre part à une date ultérieure à une délibération collective de deux à trois jours sur le problème. On envoie aux

12. Ackerman et Fishkin, 2000, p. 7.

13. Fishkin, 1991, p. 12. 
participants potentiels une trousse d'information sur le sujet de discussion « résumant les arguments en faveur des différents points de vue de manière méticuleuse et impartiale» ${ }^{14}$. Des efforts importants sont consentis afin de minimiser les obstacles entourant la participation à l'exercice de délibération à venir : on promet des honoraires et tous les coûts sont défrayés ${ }^{15}$.

La localisation de la délibération

De quatre à six semaines plus tard, les quelque trois cents participants se rendent à un point central pour prendre part au sondage d'opinion délibératif proprement dit. Cette convergence vers un point central conduit dans bien des cas les participants dans un studio de télévision, les sondages d'opinion délibératifs étant souvent enregistrés pour être télédiffusés (sur Channel 4 au R oyaume-Uni ou PBS aux États-Unis, par exemple). Les participants sont de ce fait soustraits à leur contexte quotidien et réunis dans un espace conçu pour une délibération concertée.

L'éducation des participants

Le but d'un sondage délibératif est entre autres d'accroître chez les participants la connaissance du contexte politique pertinent. C'est ainsi qu'ils obtiennent une trousse d'information les préparant à la réunion et que, durant le sondage délibératif, ils assistent à des « séances plénières au cours desquelles des spécialistes clés représentant les différents points de vue répondent aux questions formulées par les groupes de discussion ${ }^{16}$. Les participants peuvent également avoir la chance de poser des questions aux porte-parole des grands partis politiques.

Les sondages délibératifs provoquent des changements mesurables du niveau de connaissance qu' ont les participants du contexte politique en question. Par exemple:

Lors du sondage délibératif australien, la plupart des participants à l'enquête initiale ne pouvaient répondre correctement à des questions de base sur leur constitution ou sur la proposition soumise au référendum. $M$ ais au terme du week-end, ils étaient presque tous bien informés. Sur bien des questions, les pourcentages de réponses correctes étaient dans la fourchette des 80 à $90 \%$, soit une augmentation pouvant atteindre jusqu'a 57 points ${ }^{17}$.

14. Park, Alison, Jowell, R oger et $M$ cPherson, Suzi, «The Future of the $\mathrm{N}$ ational $\mathrm{H}$ ealth Service. Results from a Deliberative Poll », 1999, http ://www.la.utexas.edu/research/delpol/ papers/report_kings_fund.pdf.

15. Fishkin mesure la représentativité de l'échantillon à sa concordance avec les données du recensement le plus récent : de manière générale, de 70 à 80 pour cent des membres de l'échantillon initial prennent part au sondage d'opinion délibératif lui-même, et ce sous-ensemble tend à refléter fidèlement, tant au plan des attitudes qu'au plan démographique, I'échantillon entier. Voir Fishkin, James et Luskin, Robert, «The Deliberative Poll. A Reply to O ur Critics», The Public Perspective, janvier 1996, p. 45-49.

16. Park, Alison, Jewell, Roger et M cPherson, Suzi, 1999, p. 2.

17. Ackerman, Bruce et Fishkin, James, 2000, p. 7. Pour un survol des changements provoqués par les sondages d'opinion délibératifs, voir Center for Deliberative Polling, «D eliberative 


\section{Structurer la conversation}

Au cœur du sondage délibératif, il y a un apprentissage basé non pas simplement sur des séances d'information et des documents écrits, mais sur les échanges de raisons avec d'autres citoyens. La délibération, si on en croit Fishkin, signifie

[... ] une discussion face à face par laquelle les participants soulèvent et discutent consciencieusement des arguments adverses de façon à arriver à des jugements réfléchis sur les solutions à des problèmes publics. On peut s'interroger sur la qualité de telles délibérations à propos de : (a) l'exactitude ou la fiabilité de l'information utilisée lors des délibérations; (b) l'exhaustivité ou le manque d'exhaustivité des réponses des défenseurs des points de vue adverses aux arguments en faveur d'un point de vue donné. Tant (a) que (b) sont affaire de degré, et on peut préciser que pour qu'une délibération soit défendable, l'information doit être raisonnablement précise et les défenseurs des positions de tous bords doivent avoir un nombre significatif d'occasions de répondre aux arguments des parties adverses. Le but pour les participants, c'est d'en venir à leur propre conclusion au terme d'un examen équilibré des arguments qui s'affrontent sur la base d'une information raisonnablement bonne ${ }^{18}$.

Les participants d'un sondage délibératif passent beaucoup de temps dans des « groupes de discussion » avec un modérateur réunissant vingt participants choisis au hasard, soit des groupes assez petits pour que chacun ait, de façon significative, voix au chapitre. Les participants sont mis en contact avec des citoyens de tous horizons, ils sont incités à parler et à écouter en faisant preuve d'ouverture un vaste gamme de perspectives et à soumettre ainsi à la réflexion et la critique leurs opinions préexistantes et la vision qu'ils ont de leurs intérêts. Une telle délibération permet de filtrer et d'élever l'opinion, ouvrant des perspectives qui ont de bonnes chances, pour reprendre les termes de M ill, de combiner les points de vue de diverses vérités partielles.

\section{La conclusion de l'exercice}

À la fin du sondage d'opinion délibératif, « tous les participants remplissent à nouveau le [... ] questionnaire afin que nous puissions mesurer la direction, l'ampleur et la distribution des changements d'attitude, soit les opinions réfléchies du public par opposition à ses réactions initiales» ${ }^{19}$. A fin d'éviter les pressions sociales et par souci de symétrie, les participants remplissent aussi ce questionnaire dans le secret. C'est dire que le processus entier comporte une phase où on donne ses motifs publiquement, tandis que la mesure de l'opinion se fait sous le couvert de l'anonymat.

Polls. H ow Participants change (Selected Results) », http ://www.la.utexas.edu/research/delpol/ bluebook/delibpoll.html.

18. Fishkin, James, "The "Filter", the "M irror", and the "M ob". R eflections on D eliberative $D$ emocracy », conférence sur $D$ eliberating about $D$ eliberative $D$ emocracy, 2000 http ://www.la.utexas.edu/conf2000/papers/FilterM irrorM ob.pdf, p. 4.

19. Park, Alison, Jewell, Roger et M cPherson, Suzi, 1999, p. 2. 


\section{8 - Philosophiques / Automne 2002}

Jusqu'ici, les sondages d'opinion délibératifs ont révélé un contraste marqué entre les réponses au questionnaire au début de l'exercice et celles à la fin. On assiste non seulement à une amélioration manifeste de la connaissance des questions à l'étude, mais à des changements dramatiques dans l'opinion publique. Lors du sondage délibératif sur le système de justice pénale au Royaume-Uni, par exemple, l'accord avec la proposition voulant qu'envoyer davantage de contrevenants en prison constitue une manière efficace de combattre le crime est passé de 57 à 38 pour cent et l'appui au droit des suspects de garder le silence lors des interrogatoires de 36 à 50 pour cent; le pourcentage des répondants fortement en désaccord avec l'idée que la police devrait parfois pouvoir contourner les règles pour obtenir une condamnation est monté de 37 à 46 ; et alors que, avant délibération, 33 pour cent des participants étaient fortement opposés à ce qu'on expédie un garçon de seize ans coupable de son premier cambriolage à la prison pour adultes, après délibération, ils étaient 50 pour cent 20 .

La «voix du peuple» telle qu'elle s'exprime au terme d'un sondage d'opinion délibératif mérite désormais d'être écoutée. Si les sondages d'opinion délibératifs ne sont pas en soi des exercices de prise de décision, ils fournissent un compte-rendu de la voix du peuple en laquelle les représentants élus devraient discerner la volonté démocratique. La norme de délibération ayant été satisfaite, les décisions devraient désormais refléter celle de l'égalité politique (où les décisions reflètent les préférences des citoyens après agrégation) dans les limites que fixe la non-tyrannie.

Le modèle de Fishkin a fait face à toute une gamme de critiques et d'objections, plusieurs, entre autres, remettant en question la soi-disant ignorance des citoyens ${ }^{21}$. D'aucuns ont suggéré que c'est l'opinion publique non informée en tant que telle qui méritait de se réfléter dans la prise de décisions politiques et qu'il ne fallait pas y substituer les sondages d'opinion délibératifs $^{22}$. Enfin, les critiques se sont interrogés sur les détails de la méthodologie statistique et de la technique de sondage ${ }^{23}$. La critique que je veux explorer ici, cependant, vaut tant pour Fishkin que pour nombre de ses critiques : le travail de Fishkin est sous-tendu par une conception de l'individu comme agent, des intérêts personnels, de la connaissance de la politique et de la communication politique qui témoigne d'un point de vue particulier sur les rapports entre, d'une part, les individus et les groupes sociaux et, d'autre part, entre les groupes sociaux eux-mêmes, bref d'une ontologie sociale particulière.

20. Voir Center for Deliberative Polling.

21. Vor note 9.

22. Voir par exemple N ewport, Frank, 1996, p. 9.

23. Voir par exemple A dair, John G., "The H awthorne Effect is a Common Artifact in Social Research», The Public Perspective, janvier 1996, p. 14-16. 


\section{Trois ontologies sociales}

Une ontologie sociale décrit les unités constituant les communautés humaines, donc les rapports constitutifs entre agents individuels et groupes sociaux. Toute description des normes et des institutions démocratiques est soustendue par une certaine conception de la nature des sujets politiques et de la façon dont ils constituent des collectivités plus importantes. De ces «ontologies sociales» découlent des propositions empiriques sur la formation sociale de l'identité, les ressources épistémiques dont disposent les agents, et ainsi de suite, mais aussi des éléments philosophiques et normatifs, voire des propositions d'ordre métaphysique. Pour les fins de cet article, je décrirai trois ontologies sociales idéales-typiques : les ontologies individualiste, collectiviste et relationnelle. La liste n'est pas exhaustive et je ne prétends pas que des traits de l'un de ces types ne puissent se combiner à ceux des autres (bien que des liens internes relient ces traits et qu'ils tendent à s'agréger). C hacun de ces trois types idéaux se veut une tentative de mettre à l'avant-plan des tendances de la théorie démocratique contemporaine afin de montrer quels liens (souvent implicites) se tissent entre ontologies sociales et prescriptions touchant le processus démocratique.

Les ontologies sociales individualistes mettent les individus considérés un à un au cœur de leur description des relations sociales et politiques. Je mettrai en particulier l'accent sur les approches traitant les individus d'abord et avant tout comme des porteurs d'intérêts ${ }^{24}$. Une ontologie sociale individualiste axée sur les intérêts comprend les six affirmations suivantes :

1. Les individus sont les porteurs d'intérêts, lesquels sont à leur tour structurés et façonnés par l'environnement (y compris les autres individus et

24. L'ontologie sociale implicite de Fishkin donne une place centrale aux individus comme porteurs d'intérêts dans sa description des rapports politiques. Sur ce point, il s'accorde parfaitement avec des figures clés de diverses approches en sciences sociales de la théorie de la démocratie. Le modèle même de l'individualisme centré sur les intérêts est la description qu'a donnée Robert Dahl du «pluralisme des intérêts de groupe», dont Fishkin est manifestement tributaire. Voir Dahl, A Preface to D emocratic Theory, Chicago, Chicago University Press, 1956; Dahl, O n D emocracy, $\mathrm{N}$ ew H aven, Yale University Press, 2000. II existe un autre courant individualiste (moins pertinent pour les fins de cette discussion) qui traite les individus comme ontologiquement antérieurs aux groupes sociaux, mais qui fournit une description plus complexe de l'étiologie des buts et des fins des individus. Les individus, selon cette approche, possèdent une conception du bien, laquelle donne lieu à son tour à une conception de leurs intérêts. Pour un individualisme centré sur les conceptions du bien, voir Rawls, John, Political Liberalism, N ew York, Columbia University Press, 1993; Kymlicka, Will, Liberalism, Community and Culture, Oxford, Clarendon Press, 1991. Pour des critiques de l'individualisme comme ontologie sociale, voir Sandel, M ichael J., L iberalism and the L imits of J ustice, Cambridge, Cambridge University Press, 1983; Taylor, Charles, «Atomism», Philosophy and the H uman Sciences. Philosophical Papers, Cambridge, Cambridge University Press, 1985, vol. 2, p. 187-211. Sur la place centrale occupée par les ontologies sociales individualistes dans l'histoire de la philosophie politique libérale, voir M aclntyre, Alasdair, Whose Justice? Which R ationality?, N otre Dame, University of $N$ otre Dame Press, 1988; Taylor, Charles, Sources of the Self. The M aking of the M odern Identity, Cambridge, M ass., H arvard University Press, 1989. 
les agrégats d'individus). N os intérêts demeurent relativement stables avec le temps, bien que notre aptitude à comprendre quels sont nos intérêts et la nature des actions et politiques les plus susceptibles de les servir puisse varier. Dans la mesure où l'identité des individus (leurs désirs, leurs valeurs, la compréhension qu'ils ont d'eux-mêmes) sont un trait saillant de la politique, cela donne lieu à des constellations d'intérêts passablement stables.

2. Les individus sont ontologiquement antérieurs aux groupes, lesquels sont des agrégats d'individus ${ }^{25}$. Les groupes partageant les mêmes intérêts, opinions ou valeurs peuvent se décomposer en fonction des individus les constituant.

3. Qui dit politique dit mobilisation des individus autour d'intérêts communs en vue de faire progresser ces intérêts au plan politique ${ }^{26}$.

4. Le pouvoir exerce des contraintes sur les intérêts mais ne les constitue pas. La politique, comprise comme une lutte des individus et des groupes en vue de satisfaire leurs intérêts, implique la mobilisation du pouvoir. Si les rapports de pouvoir jouent un rôle important dans la capacité des individus à satisfaire leurs intérêts, ces derniers préexistent néanmoins à l'exercice du pouvoir.

5. Les mondes social et matériel sont en principe cognitivement accessibles à tous. La politique est aussi une lutte pour l'accès à la connaissance : l'impression que l'on a de la meilleure manière de satisfaire ses intérêts et de leur situation parmi les intérêts des divers individus et groupes dépend de manière cruciale de la conception que l'on a des intérêts d'autrui et de son appréhension des liens de cause à effet. Suivant une description individualiste, la connaissance des intérêts et des liens de cause à effet peut être inégalement distribuée entre individus et groupes, mais l'appartenance à un groupe ne façonne la connaissance que de manière contingente.

6. Cette ontologie sociale a des dimensions empiriques et normatives. Empiriquement parlant, nous sommes des individus porteurs d'intérêts, notre engagement en politique consistant à regrouper nos intérêts. En termes normatifs, les bonnes institutions démocratiques font un arbitrage équitable entre intérêts en s'assurant que les décisions collectives reflètent une bonne compréhension des divers intérêts en jeu et ne marginalisent pas systématiquement les intérêts de quelque groupe particulier que ce soit.

25. Sandel, 1983 a donné une formulation de cette thèse de l'antériorité ontologique qui a fait date. Voir aussi Young, Justice and the Politics of D ifference.

26. C'est dire que je vois des liens étroits entre ontologies sociales individualistes et pluralisme des groupes d'intérêts comme description de la représentation, liens qui apparaissent clairement dans l'œuvre de Fishkin. Voir Williams, 1988, p. 64-70 ; Dahl, 1956. 
L es ontologies sociales collectivistes. Chez les penseurs politiques récents, les critiques les mieux connues des ontologies sociales individualistes, et qui se veulent le plus souvent une solution de rechange à ces ontologies, ont été communautariennes ou, plus largement, «collectivistes». Si les approches collectivistes constituent un contrepoids important à l'individualisme (tout particulièrement dans les débats entre libéralisme et communautarisme, au cours des dernières décennies), cette dichotomie s'est à ce point enracinée que les défauts du collectivisme (tels que sa propension à exagérer I'homogénéité interne des groupes ou le trop peu d'attention qu'il accorde aux rapports de pouvoir) sont trop volontiers imputés à des critiques par ailleurs distinctes de l'individualisme. Aussi, bien que les ontologies sociales individualistes et relationnelles occuperont une place de premier plan dans la discussion de Fishkin ci-dessous, on se fera une image plus nette de ces deux types si on leur y oppose un troisième, le collectivisme. Les ontologies sociales collectivistes considèrent les individus comme profondément constitués par leur appartenance à un groupe. C'est dire que les conceptions et l'identité qu'ils partagent occupent une place centrale dans l'interaction sociale et politique ${ }^{27}$. Ainsi :

1. Les groupes définissent nos horizons épistémique et normatif. Suivant les descriptions collectivistes, les identités personnelles, les conceptions et les valeurs se constituent au sein de communautés de croyances et de pratiques. C'est là une affirmation d'ordre empirique - les individus opèrent toujours avec les ressources linguistiques et épistémiques que leur communauté met à leur disposition - et normatif - les êtres humains, dit-on, s'épanouissent quand ils s'insèrent de façon stable dans une communauté partageant les mêmes pratiques et les mêmes significations. L'appartenance à une communauté constitutive donnée produit une identité d'un genre particulier avec ses intérêts correspondants. C'est dire que les perspectives du groupe et ses intérêts sont relativement stables dans le temps.

2. Les groupes sont ontologiquement antérieurs aux individus. Ceci découle du point précédent : les individus sont produits par socialisation dans un contexte particulier. En effet, le concept même d'«individu» témoigne d'un contexte historique et culturel particulier ${ }^{28}$.

27. Le communautarisme est le courant de philosophie politique où le collectivisme se manifeste de la manière la plus intéressante. Voir Sandel, M ichael, 1983; M aclntyre, Alasdair, After Virtue. A Study in M oral Theory, N otre Dame, University of N otre Dame Press, 1981; $M$ aclntyre, 1988. En décrivant cette ontologie, je suis prêt à concéder que les collectivistes disent reconnaître à la fois la complexité de la construction sociale étant donné les multiples appartenances de groupe et les rapports de pouvoir dans la constitution des groupes sociaux. Ce qui éloigne les collectivistes d'une ontologie sociale relationnelle, c'est le peu de place qu'occupe la reconnaissance de ces faits dans leur discussion générale, en particulier dans les généralisations qu'ils énoncent sur les conceptions communes et les collectifs sociaux.

28. Voir Taylor, 1989. 
3. La politique est une lutte au sein des communautés constitutives et entre ces mêmes communautés. La mobilisation politique met en jeu les diverses communautés constitutives, lesquelles ne se distinguent pas seulement en fonction de leurs intérêts, mais aussi de leurs perspectives épistémiques, de leurs engagements normatifs et de leur identité. Ici, les dimensions descriptive et normative des ontologies sociales collectivistes tendent à diverger, puisque les idéologies et institutions dominantes dans les États libéraux démocratiques contemporains donnent indéniablement la place centrale aux intérêts et aux revendications de droits individuels : les collectivistes souscrivent à cette affirmation empirique, mais ils y voient une lacune des institutions politiques contemporaines. Les communautariens considèrent tant l'individualisme que le pluralisme des valeurs comme problématiques, et les idéaux qu'ils construisent visent à des politiques témoignant d'une meilleure intégration sur le plan normatif 29 .

4. Les collectivistes reconnaissent volontiers qu'il y a une lutte entre les groupes et au sein des groupes, mais ils ne sont pas enclins à considérer les conceptions et valeurs distinguant les groupes particuliers comme le produit de rapports de pouvoir entre les divers groupes. Ils tendent plutôt à considérer que ces pratiques et conceptions communes se développent de manière organique et changent suivant une logique interne $\mathrm{e}^{30}$.

5. L'appartenance à un groupe constitue son horizon épistémique. Les groupes se définissent selon l'horizon de la conception qu'ils ont du monde matériel et les questions d'ordre normatif. Ainsi, les ontologies sociales collectivistes tendent à épouser une certaine forme de relativisme moral, s'attendent à ce que des gouffres épistémiques séparent les différents groupes sociaux et sont sceptiques vis-à-vis de la commensurabilité des perspectives propres aux groupes ${ }^{31}$.

6. Cette ontologie sociale a des dimensions empiriques et normatives. Comme je l'ai déjà noté, les ontologies sociales collectivistes tendent à critiquer l'hégémonie de l'individualisme dans la théorie et la pratique politique libérales. Les collectivistes mettent l'accent sur l'inexactitude au plan empirique de l'individualisme - car nous sommes en fait profondément constitués par notre appartenance à une communauté mais ils déplorent aussi les distorsions importantes que l'individualisme

29. Cela est particulièrement vrai de l'œuvre de M aclntyre et émerge clairement de celle de Sandel.

30. M acIntyre, 1988 incarne bien ce traitement de la logique interne des traditions de pratique et de questionnement.

31. Les analyses de la commensurabilité des conceptions du monde et des conditions dans lesquelles on peut établir la rationalité de ces conceptions sont une dimension chargée et intéressante de la littérature. Voir par exemple Taylor, Charles, sur la «fusion des horizons» dans M ulticulturalism and «The Politics of Recognition», Princeton, Princeton University Press, 1992; M aclntyre, 1988. 
a imposées à notre monde politique pour qu'il soit à son image. Ainsi, les collectivistes proposent une vision normative de la politique qui soit plus attentive à la valeur et la saillance politique des appartenances de groupe.

Les tenants du collectivisme font valoir - et je suis d'accord avec eux que cette ontologie sociale marque un progrès par rapport à l'individualisme. Pour commencer, il semble qu'il y ait de puissants arguments en faveur de la thèse voulant que sa description des relations entre individu et groupe soit plus plausible sur les plans philosophique, sociologique et anthropologique. (Défendre cette assertion me ferait largement déborder du cadre de cet article) ${ }^{32}$. Ensuite, le collectivisme a raison de voir dans l'individualisme lui-même une conception des agents propreà un contexte culturel et historique et, de manière corollaire, il révèle comment la naturalisation de cette conception des agents constitue un geste politiquement chargéz3.

L es ontologies sociales relationnelles considèrent les individus comme profondément tributaires de leur appartenance à des groupes sociaux, mais elles mettent aussi l'accent sur la pluralité, la complexité et le caractère mutuellement constitutif de telles appartenances ${ }^{34}$.

1. Les groupes s'entrecroisent, définissant les horizons épistémiques et normatifs de leurs membres. Comme les descriptions collectivistes, les ontologies sociales relationnelles considèrent que les identités, les conceptions et les valeurs des individus se constituent au sein de communautés de croyances et de pratiques. Cependant, les descriptions relationnelles soulignent la multiplicité des groupes auxquels appartient toute personne et la façon dont le poids des différentes appartenances varie selon des modalités complexes en fonction du contexte. La conception qu'on se fait de son identité et de ses intérêts est façonnée par les expériences spécifiques à un groupe, mais elle est aussi susceptible de se négocier et de changer au fil des passages d'un contexte socio-politique à l'autre.

2. Les groupes sont ontologiquement antérieurs aux individus. Étant donné la complexité des appartenances, cependant, cette antériorité ne permet

32. Pour des arguments en ce sens, voir Walzer, M ichael, Interpretation and Social Criticism, Cambridge, M ass., H arvard University Press, 1987; Stout, J effrey, Ethics after Babel. The $L$ anguages of M orals and their D iscontents, Boston, Beacon Press, 1988 ; Taylor, Charles, 1989.

33. Pour une argumentation plus étendue sur ce point, voir Kahane, David (à paraître), «Alternative Dispute and the Politics of Cultural Generalization», $\mathrm{H}$ arvard J ournal of Negotiation.

34. Cette perspective (que je fais mienne) a des liens avec la pensée continentale et « postmoderne», mais ses manifestations les plus claires se trouvent chez des théoriciens nord-américains. Voir en particulier Connolly, William E., The Ethos of Pluralization, M inneapolis, University of M innesota Press, 1995, mais aussi Butler, Judith, G ender Trouble. Feminism and the Subversion of I dentity, N ew York, Routledge, 1990; Wendy Brown, States of I njury. Power and Freedom in L ate M odernity, Princeton, Princeton University Press, 1995. 
pas de prédire si facilement quels sont leur identité et leurs intérêts. La communauté d'identité et d'intérêts se reconstruit sans cesse.

3. La politique implique la mobilisation et la construction d'identités fondées sur les groupes. Elle mobilise l'identité de groupes existants, mais elle forme et consolide aussi de nouveaux groupes et identités ${ }^{35}$. C'est dire que les ontologies sociales relationnelles se démarquent des ontologies sociales collectivistes, qui traitent implicitement les groupes comme des entités statiques et hermétiques.

4. Le pouvoir construit tant les groupes que les individus. Les ontologies sociales relationnelles mettent l'accent sur la façon dont les groupes sociaux se définissent à travers leurs rapports de pouvoir. De tels rapports entre groupes jouent sur la formation des identités fondées sur des groupes selon la dynamique d'attribution, de coercition, de marginalisation et d'exploitation par rapport aux autres groupes ${ }^{36}$. Si I'appartenance fondée sur les groupes est en principe fluide et multiple, les jeux de pouvoir peuvent faire de l'attribution d'une certaine appartenance une source d'inconvénients durables, cette dynamique étant d'une pertinence particulière pour les normes de représentation démocratique ${ }^{37}$. La dynamique du pouvoir au sein des groupes joue également un rôle clé en ce qu'elle façonne la représentation politique que les groupes se font d'eux-mêmes (ce qui implique souvent qu'on minimise l'hétérogénéité interne). Enfin, les ontologies sociales relationnelles (s'inspirant souvent en cela de Foucault) tendent à concevoir le pouvoir - tant au sein des groupes qu'entre groupes - comme à la fois fécond et répressif, puisque celui-ci marque toutes les relations entre agents au lieu de s'exercer de manière souveraine depuis un lieu unique. Étant donné ce jeu de pouvoir au sein des groupes et entre groupes, il est particulièrement périlleux de réifier les appartenances à un groupe ou les conceptions qui y sont attachées, celles-ci étant toujours fluctuantes et se négociant en fonction des différences entre pouvoir individuel et pouvoir de groupe.

5. Les «relationnalistes» soulignent la complexité de la communication et l'importance des différences de pouvoir entre groupes dans les conceptions qu'on se fait d'autrui. L'appartenance à un groupe social implique souvent des expériences communes, d'où une perspective commune; c'est particulièrement vrai de ceux auquel on attribue une appartenance à des groupes relégués à la marge. Par ailleurs, la complexité des appartenances et de la formation de l'identité rend périlleuses les généralisations sur cette communauté d'expériences et de perspectives et suggère

35. Voir Connolly, 1995.

36. Voir Kahane, David, «Diversity and Civic Solidarity», Journal of Political Philosophy, vol. $7, n^{0} 3$, p. 243-262.

37. Voir Williams, 1998. 
qu'inévitablement les individus sont jusqu'à un certain point opaques aux yeux d'autrui. Cette opacité entre soi et les autres, cependant, n'est pas également distribuée : la place qu'on occupe au sein des rapports de pouvoir basés sur les groupes et les privilèges influe sur sa connaissance des membres des autres groupes sociaux et sur l'étendue de la connaissance d'autrui qu'on tend à s'attribuer. Bref, les membres des groupes opprimés ont de puissants incitatifs à comprendre leurs oppresseurs tandis que l'inverse est moins vrai, de sorte que les privilégiés sont plus enclins que les autres à considérer leur perspective comme la norme ${ }^{38}$. Ce dernier point sur la normalisation des points de vue privilégiés donne lieu, dans les ontologies sociales relationnelles, à une attitude critique vis-à-vis des prétentions à l'autorité épistémique, en particulier vis-àvis des prétentions à la qualité d'expert ou à la neutralité. II donne également lieu à une attitude tout aussi critique vis-à-vis des assertions sur les modes de communication «raisonnables» et «déraisonnables» 39 .

6. Cette ontologie sociale a des dimensions empiriques et normatives. Une ontologie sociale relationnelle prétend décrire notre constitution sociale et la complexité de nos appartenances et de nos identités. $M$ ais elle entraîne aussi une vision normative de la politique démocratique qui reconnaît les appartenances de groupes - et les rapports de pouvoir entre groupes - sans les réifier ${ }^{40}$.

Cette ontologie sociale préserve les progrès faits par le collectivisme sur l'individualisme - étant consciente que ce sont les groupes qui constituent la société et reconnaissant la spécificité culturelle de l'individualisme comme norme de la notion d'agent - mais elle surmonte les embûches auxquelles se heurte le collectivisme. Pour commencer, I'approche relationnelle est encore plus plausible sur le plan social et anthropologique, dans la mesure où elle s'accommode des appartenances plurielles et de l'hétérogénéité interne des groupes. Les collectivistes, pour leur part, prêtent le flanc aux accusations d'essentialisme tant dans leurs descriptions des groupes sociaux que dans leur idéalisation au plan normatif des communautés organiquement structurées ${ }^{41}$. Ensuite, on peut dire, à la lumière des considérations qui précèdent, qu'une ontologie sociale relationnelle s'accommode bien de la complexité et

38. Cette analyse est à l'évidence redevable à l'épistémologie du point de vue féministe. Voir entre autres H arding, Sandra, «R ethinking Standpoint Epistemology. "W hat is Strong O bjectivity?" », dans A Icoff, L. et Potter, E., dir., Feminist Epistemologies, N ew York, Routledge, 1993, p. 49-82. Pour une application de cette analyse aux rapports entre hommes et femmes, voir Kahane, David, «M ale Feminism as Oxymoron», dans Digby, T., dir., M en doing Feminism, $\mathrm{N}$ ew York, Routledge, 1998, p. 213-236.

39. Là-dessus, voir Young, 2000, chap. 2.

40. Voir Brown, 1995 et Connolly, 1995.

41. De tous les collectivistes cités ici, $M$ aclntyre est celui qui prête le plus le flanc à cette critique. Voir Clark, Stephen R. L., «M orals, M oore, and M aclntyre», Inquiry, vol. 26, n 4, 1983, p. 424-445. 


\section{6 - Philosophiques / Automne 2002}

de l'opacité des identités, aspect forcément minimisé par les ontologies collectivistes. Enfin, I'approche relationnelle parle explicitement du pouvoir social tant au sein des groupes qu'entre groupes et décrit comment les groupes se façonnent les uns les autres du fait des asymétries de pouvoir, alors que les ontologies sociales collectivistes tendent à minimiser la co-formation des groupes sociaux et la place du pouvoir dans cette co-formation.

Les ontologies sociales relationnelles, cependant, soulèvent de nouvelles difficultés : elles font en sorte qu'il est périlleux d'énoncer des généralisations sur les groupes sociaux, leurs conceptions et leurs limites, et cela a des implications sur le processus démocratique. J'y reviendrai. Je pars cependant de la prémisse que ni les ontologies individualistes ni les ontologies collectivistes ne rendent compte adéquatement de notre identité, de nos liens d'appartenance et de nos intérêts à des fins politiques, de sorte qu'il ne nous reste plus qu'à composer avec la complexité des descriptions relationnelles.

Pour le moment, cependant, tournons-nous vers l'analyse que fait Fishkin des pathologies politiques et le remède qu'il y apporte, les sondages d'opinion délibératifs. M on intention est de faire valoir qu'il s'appuie implicitement sur une ontologie sociale individualiste, laquelle sous-tend un certain nombre de tensions et d'apories dans sa description.

\section{L'individualisme de Fishkin : critique}

N ous pouvons commencer à apprécier jusqu'à quel point l'analyse de Fishkin penche pour une ontologie sociale individualiste en examinant son analyse des déficits démocratiques contemporains. On se rappellera que ce qui préoccupe Fishkin, c'est la façon dont les aspects égalitaires de la démocratie ont pris le pas sur ceux qui sont liés à la délibération, si bien que les décisions reflètent les opinions non informées et irréfléchies des citoyens. Fishkin n'est pas indifférent à l'influence des appartenances de groupe sur cette dynamique. II explique par exemple comment l'accès à la richesse influe sur l'accès aux médias, violant de ce fait les conditions d'isolement. Pourtant, à un niveau plus large, Fishkin aborde l'ignorance et le retrait de la sphère politique comme s'il s'agissait de traits relativement indifférenciés dans la population considérée comme une masse. II suggère en effet que les " apathiques positifs», les «politiquement impuissants» et les «court-circuités» se distribuent également parmi les groupes sociaux et que leur aliénation vis-à-vis de la politique procède de raisons similaires ${ }^{42}$. Pour des raisons d'ordre social, politique et culturel, nous dit-on, l'engagement et les opinions politiques sont aujourd'hui dévalorisés aux États-Unis. Dans ce contexte, le défi est tout simplement de conduire divers citoyens à parler, réfléchir et se faire des opinions mieux informées.

Ce portrait des déficits démocratiques a son versant positif : la démocratie devrait faire en sorte que la voix du peuple vaille la peine d'être écoutée.

42. Fishkin, 1991, p. 57. 
$M$ ais de qui est-ce la voix? Le développement précédent sur les ontologies sociales met au jour la façon dont Fishkin considère la voix du peuple comme l'agrégation des voix individuelles faisant part de leurs préférences et leurs intérêts dans un contexte où sont prises au sérieux les perspectives de tous les groupes d'intérêts importants et où les minorités ne sont pas systématiquement marginalisées. Cela se voit clairement, par exemple, à sa justification de la délibération politique. La délibération est un élément clé de la démocratie dans la mesure où elle expose les citoyens aux perspectives d'autrui tout en les poussant à clarifier leurs propres intérêts. Q u'on se rappelle comment Fishkin invoque $\mathrm{D}$ ahl : «A fin d'exprimer de manière précise leurs préférences, tous les citoyens et citoyennes doivent disposer d'occasions égales et adéquates pour découvrir et confirmer, le temps qu'il faut pour prendre la décision, quelles sont leurs préférences concernant le point à trancher ${ }^{43}$. Les sondages d'opinion délibératifs offrent un contexte où une coupe transversale représentative réunit des citoyens pouvant clarifier leurs propres préférences et apprendre quelles sont celles d'autrui tout en comprenant mieux dans quel cadre empirique se décide telle politique particulière. Ce faisant, ils ne sont plus confinés à leurs propres intérêts étroitement compris, leur compréhension inadéquate des liens de cause à effet en jeu et leurs valeurs non clarifiées, puisqu'ils acquièrent une compréhension élargie de leurs intérêts et des questions politiques. Divers individus passent donc de concert à une appréhension commune et plus précise des enjeux politiques, de la gamme des opinions au sein de la société et des politiques appropriées pour y répondre.

On observera comment cet invidual isme joue à chacune des étapes du sondage d'opinion délibératif que nous avons décrites ci-dessus et comment il conduit à des lacunes et des problèmes mis en lumière par les autres ontologies sociales. Dans les pages qui vont suivre, je me servirai de l'exemple du sondage délibératif britannique sur le système pénal qui s'est déroulé en $1994^{44}$. J e me concentrerai d'abord sur les difficultés que soulève le processus prévu par Fishkin. Dans une section ultérieure, je me pencherai sur les solutions qui pourraient y être apportées.

\section{La sélection des participants}

Les sondages d'opinion délibératifs, dit Fishkin, utiliseraient les «techniques d'échantillonnage mises au point par la recherche sur I'opinion publique pour représenter et réunir une version de ce que nous sommes tous» ${ }^{45}$. Sa notion de représentation adéquate exige donc une coupe transversale de la population constituée de manière aléatoire : l'issue du sondage délibératif traduit de

43. I bid., p. 36 .

44. Pour une brève discussion de ce processus, voir Fishkin, 1995, p. 165-168. Voir aussi Institute of G overnance, The Citizen Engagement R ound Table. D eliberative Polling (17 avril), http ://www.iog.ca/publications/cert1.pdf, 1996, p. 3. Pour un résumé des résultats du sondage, voir Fishkin, 1995, p. 177-181.

45. Fishkin, 1991, p. 12. 


\section{8 - Philosophiques / Automne 2002}

manière significative les opinions de la communauté dans son ensemble en vertu de sa représentativité, en plus d'avoir pour effet d'élever la délibération. $M$ ais dans sa justification du recours à une coupe transversale, Fishkin s'appuie sur l'image qu'il donne de la non-participation comme d'un phénomène générique et non pas d'un phénomène liéà des appartenances et des attributions de groupe particulières sur fond d'inégalité des pouvoirs et des opportunités. Son objet est de réunir des citoyens chez qui l'apathie serait un phénomène généralisé afin qu'ils puissent se forger des préférences de manière informée.

En discutant de la condition d'égalité, Fishkin parle de l'importance d'assurer que « toute la gamme des intérêts ayant un nombre significatif de partisans» soit effectivement entendue ${ }^{46}$. Q uand on apprécie la saillance des appartenances de groupe conçues comme plus que la simple convergence d'intérêts, on voit à quel point les longues histoires de marginalisation sont significatives. Ainsi, même dans les termes de l'approche statistique de Fishkin, il est permis de se demander si un échantillon d'une taille donnée inclura effectivement tous les groupes d'intérêts significatifs du point de vue de l'égalité démocratique. Des groupes relativement petits peuvent avoir des prétentions urgentes à l'inclusion en raison de l'oppression, l'exclusion politique ou la marginalisation dont ils ont longtemps fait l'objet ${ }^{47}$.

II se peut qu'on doive explicitement inclure les membres de groupes spécifiques dans les processus de délibération en raison de l'identité des personnes touchées au premier chef par une politique en particulier. Considérons le sondage d'opinion délibératif sur la réforme du système de justice pénale britannique : certains groupes paraissent plus directement concernés par une telle législation que d'autres, les groupes surreprésentés dans la population carcérale en raison de leur race ou de leur revenu, par exemple. Fishkin se penche sur la suggestion de Burnham qui veut qu' on puisse mettre en place des processus délibératifs utilisant des échantillons représentant «les intérêts touchés dans divers secteurs [qui] peuvent être déterminés à la lumière de diverses données obtenues par sondage ou du jugement des institutions concernées ${ }^{48}$. Fishkin rejette cette méthode de sélection : il utilise une coupe transversale de l'électorat entier plutôt qu'un échantillon des personnes spécifiquement affectées par des sujets particuliers ${ }^{49}$.

46. Ibid., p. 33.

47. Comme Fishkin le note lui-même, dans une analyse individualiste, «il y a énormément de place à un débat sur ce qui peut être considéré comme une opinion ralliant un "nombre significatif" de personnes et sur ce ce qui peut être considéré comme un "intérêt intense" chez ceux qui soutiennent une telle opinion ». (Fishkin, 1991, p. 33-34.)

48. Ibid., p. 98.

49. Les échantillons de la population en vue des sondages d'opinion délibératifs se veulent suffisamment grands pour fournir un microcosme de la population entière en question. Ainsi, dans cet exemple, le fait est que les 869 citoyens sélectionnés de manière aléatoire « représentaient de près la nation par l'âge, la classe, la représentation géographique, le sexe et le degré d'instruction ». (Fishkin, James, «Ideal Citizens give a Considered Judgment», The Independent, 9 mai 1994, p. 8.) 
En attendant la délibération

M ême du point de vue des ontologies sociales non individualistes, la méthode de Fishkin pour sélectionner les participants ne comporte pas nécessairement de défauts irrémédiables : il se peut qu'elle parvienne à réunir des membres de groupes sociaux clés même si on n'a pas mis explicitement l'accent sur de tels groupes. $M$ ais son individualisme a-t-il un impact sur ce qu'il prévoit qu'il se passera dans l'intervalle séparant la sélection et l'enquête initiales et le sondage délibératif lui-même?

D'aucuns sont préoccupés par l'élitisme du processus, élitisme dont on pourrait faire val oir qu'il découle d'une ontologie sociale individualiste. Q u'on se rappelle que des trois types idéaux décrits plus haut, seul l'individualisme implique une épistémologie en vertu de laquelle les contraintes sur le savoir qu'exercent les appartenances de groupes ne sont que contingentes : s'il est vrai que les individus expriment généralement les points de vue typiques de ceux qui sont placés dans les mêmes circonstances, il leur est possible de les transcender. Étant donné la description que fait Fishkin de la distribution de l'ignorance parmi les citoyens, on voit mieux d'où lui vient sa foi en la capacité des experts à s'instruire d'eux-mêmes et à développer une perspective qui ne soit pas significativement marquée par la situation sociale qu'ils se trouvent à occuper ${ }^{50}$. Si Fishkin opère implicitement dans une telle perspective ontologique et épistémologique, on comprend mieux qu'il soit assuré de la pertinence de distribuer une trousse d'information sur les problèmes à discuter dans le cadre du sondage d'opinion délibératif, trousse « résumant les arguments en faveur des différents points de vue de manière méticuleuse et impartiale» ${ }^{51}$. Selon une perspective collectiviste ou relationnelle, en revanche, l'élaboration d'une telle trousse d'information par un individu ou groupe issu de l'élite risque de reproduire la perspective de son groupe social aux dépens des autres, même si le but avoué est de présenter une gamme respectable d'opinions et d'interprétations des faits. Parmi la pléthore de sources d'information sur le système de justice pénale britannique, que faire entrer dans la trousse d'information? Les différentes conceptions de la manière dont la perspective influence (légitime) I'opinion peuvent dicter l'élaboration d'une trousse contenant : les résultats de divers travaux en sciences sociales et les énoncés de politiques des divers partis; des recommandations dues aux divers lobbys ou aux groupes de défense des droits des détenus; des documents pondus par des anarchistes ou des radicaux d'obédience foucaldienne exigeant le démantèlement du complexe répressif; des témoignages de détenus, etc. En raison de la pléthore de documents possibles, il faut une certaine sélection, laquelle ne peut jamais être «impartiale», comme le voudrait Fishkin, mais représente une certaine interprétation de ce qui constitue une connaissance valable du problème.

50. Pour les liens entre cette épistémologie et le pluralisme des groupes d'intérêts, voir Williams, 1998, p. 138-139.

51. Park, Alison, J owell, Roger, et M cPherson, Suzi, 1999. 


\section{0 - Philosophiques / Automne 2002}

Toujours au sujet de l'intervalle séparant la sélection des participants et le sondage délibératif, on peut se demander en quoi les participants «représentent» un intérêt ou un groupe. Si, à en croire Fishkin, les participants ont de bonnes chances de ne rien connaître aux sujets sur lesquels ils seront sondés, le soin avec lequel ils sont sélectionnés suggère que chacun apportera quelque chose de bien différent à la délibération : les expériences, opinions et intérêts typiques de ceux qui occupent la même place que lui dans la société. Suivant la description individualiste, la place qu'on occupe dans la société décide des circonstances qui conditionnent généralement ses intérêts; selon la description collectiviste, la place qu'on occupe dans la société décide de l'horizon des conceptions façonnant à leur tour sa perspective politique, y compris l'impression qu'on a de ce que sont ses propres intérêts : suivant l'une ou l'autre conception, donc, un participant est d'emblée équipé pour « représenter » toux ceux qui occupent la même place que lui dans la société, qu'il soit Anglo-Saxon, ou immigrant de la première génération, ou femme, ou exdétenu. Ses intérêts sont conditionnés par les expériences spécifiques à son groupe, et donc communs aux autres membres du groupe et relativement stables. Les choses sont plus complexes pour une ontologie sociale relationnelle, où les intérêts spécifiques à un groupe et ses conceptions ne sont pas inhérents aux individus mais se reconstruisent au fil des interactions socio-politiques. Selon cette perspective, aucun Britannique noir, par exemple, ne « représente» le groupe des Britanniques noirs ou ses intérêts de manière tout à fait prévisible; pour que ce groupe soit représenté, il faudra que les membres du groupe aient auparavant interagi durablement sur le plan politique, y compris sur la question même de savoir quelle est la manière adéquate de le représenter.

\section{La localisation de la délibération}

Un sondage délibératif sélectionne les participants de manière aléatoire et non pas en fonction de leur appartenance de groupe, et c'est à titre individuel qu'on leur offre la trousse d'information assemblée par des membres de l'élite. Le sondage détache alors les participants de leur réseau en vue de plusieurs journées de délibération. Cela a un impact, dans la mesure où nos appartenances de groupes et nos relations avec autrui nous façonnent perpétuellement. Aussi les doutes que nous soulevions au sujet de l'intervalle précédant le sondage d'opinion délibératif persistent-ils s'agissant de la structure du sondage lui-même. Les deux personnes dans l'échantillon qui se sont révélées avoir un dossier criminel, par exemple, en sont venues à représenter ce point de vue social pour tous les participants, et pourtant, elles I'ont fait sans avoir de contact ou négocier avec le groupe qu'elles étaient censées représenter.

\section{L'éducation des participants}

Les problèmes relatifs à l'élitisme et au contrôle de l'information perdurent égal ement lors du sondage délibératif lui-même. Durant les délibérations sur le système de justice pénale britannique, par exemple, quels étaient les critères 
déterminant qui agirait à titre d'autorité ou de personne-ressource lors des séances plénières? Là encore, on peut voir les effets d'une conception de la délibération voulant qu'il s'agisse d'un processus générique de collecte de renseignements. $\mathrm{M}$ ais il apparaît clairement que ceux qui sont invités à s'adresser aux participants ont leur programme, leur idéologie et leurs partipris en plus de se distinguer par le profil propre à leur groupe - éducation, classe, race, etc. Le processus de délibération de Fishkin ne met pas en lumière ces aspects de sa propre constitution en vue d'un examen démocratique. II préserve la hiérarchie entre organisateurs et participants en plus d'instaurer un rapport particulier entre experts invités et participants.

J'aimerais suggérer que l'indifférence de Fishkin pour la façon dont les identités propres aux groupes, les affinités et les inclinations peuvent biaiser les délibérations dérive d'une conception individualiste de la délibération ellemême. Fishkin décrit un paysage politique où la délibération publique s'est pratiquement tarie : les gens votent et se font des opinions politiques sur la base d'une compréhension superficielle des problèmes en jeu sans profiter du point de vue d'autrui. Cette opposition lui fournit une excuse pour donner un grand poids à ce dernier aspect : le seul fait qu'il ait su créer un cadre où les participants sont conduits à prendre connaissance des faits, entendre le point de vue d'autrui et bien réfléchir constitue une contribution importante.

\section{Structurer la conversation}

Fishkin ne fournit pas beaucoup de détails sur la structure de la délibération proprement dite. II est vrai qu'il précise que «les participants délibéreront de manière interactive au sein d'un petit groupe, chacun fera la connaissance des autres et on s'attend que tous prennent leur décision au terme de discussions et de débats communs $\$ 52$. Tous les participants se rassemblent pour des périodes de questions qu'ils soumettent aux candidats et aux experts ${ }^{53}$. Et la «décision» ultime prend non pas la forme d'un vote ou d'un consensus, mais d'un sondage d'opinion. Q uel genre de réflexion et de compréhension mutuelle un tel échange peut-il susciter?

Fishkin s'attend pour sa part à ce que les opinions des participants sur les problèmes débattus gagnent en cohérence :

$\mathrm{N}$ ous ne savons pas à quel point les sondages d'opinion délibératifs contribueraient à la formation d'opinions réfléchies. Cependant, il semble qu'il y ait

52. Fishkin, 1991, p. 93.

53. Fishkin précise que lors du sondage d'opinion délibératif sur le système de justice pénale, des discussions en petits groupes se sont déroulées tôt au cours du processus, afin de jouer un rôle d' « activation ». Le but de cette séquence est de « faciliter au départ la fusion des groupes, afin d'identifier d'emblée leurs préoccupations clés, créer des rapports entre eux, établir le programme des questions et des préoccupations qu'ils veulent soulever, pour être mis en contact après seulement avec les divers experts et hommes et femmes politiques de toutes tendances. [Ce] modèle, au lieu de reprendre platement le programme des experts, dynamise une voix publique venant des citoyens afin qu'elle s'adresse aux élites». (Fishkin, 1995, p. 172.) 


\section{2 · Philosophiques / Automne 2002}

de bonnes chances que bien des [... ] non-attitudes ou pseudo-opinions soient remplacées par des points de vue plus rationnels et mieux étayés et qui soient peut-être plus cohérents avec d'autres éléments du système de croyances du répondant ${ }^{54}$.

Ces reformulations viennent de ce que les participants sont appelés à énoncer et défendre leur point de vue devant autrui et de ce qu'ils se font une meilleure idée de la structure des points de vue adverses. Fishkin suggère en outre que la compréhension mutuelle découlant de la délibération au sein de petits groupes fait décroître la polarisation, dans la mesure où les positions extrêmes reposent sur des erreurs cognitives, une image caricaturale des adversaires et des incohérences internes.

Étant donné ce portrait de la formation de l'opinion, il semble qu'il y ait trois façons dont la délibération peut décevoir nos attentes : (i) toute la gamme des arguments pertinents n'est pas soumise à l'attention des participants; (ii) les participants n'ont pas suffisamment d'occasions de répondre aux arguments d'autrui ; (iii) «les participants ne disposent pas de toute la connaissance ou de toutes les aptitudes qui leur permettraient de comprendre les arguments exprimés en faveur de l'une ou l'autre position $\gg 55$. Un certain nombre d'objections contre la structure du sondage d'opinion délibératif de Fishkin sont liées à un troisième point : la somme des connaissances et aptitudes requises des participants pour qu'ils comprennent les positions d'autrui. Si on conçoit la délibération comme un processus de collecte de renseignements par des individus dont les intérêts diffèrent, tout ce dont ceux-ci ont besoin, c'est suffisamment de temps pour se parler. M ais j'ai fait valoir qu'il nous faut apprécier le fait que les appartenances de groupe, définies dans des contextes de différences de pouvoir, ne façonnent pas seulement les intérêts des participants, mais leur identité, leur horizon interprétatif, leur style de communication, les domaines où ils sont ouverts et ceux auxquels ils ne connaissent pas grand-chose. Ces connaissances et aptitudes spécifiques au groupe nous montrent quels sont les défis que doivent relever la communication et la délibération, défis que Fishkin à bien des égards laisse de côté.

\section{La conclusion de l'exercice}

$C^{\prime}$ est un point central de la méthode de Fishkin que le sondage d'opinion délibératif doive se conclure avec le même questionnaire qu'à l'étape initiale. Le but de l'exercice est de mesurer les changements d'opinion du début à la fin, les opinions après délibération devant être considérées comme le portrait de ce que penserait le public en général s'il était réfléchi et adéquatement informé. Là encore, la conclusion de l'exercice met l'accent sur la délibération comme collecte de renseignements par les individus : ce qui importe, c'est l'exposition à des renseignements d'ordre empirique et à d'autres points de

54. Fishkin, 1991, p. 83.

55. Ibid., p. 37. 
vue, de façon qu'on puisse réviser son impression sur les politiques susceptibles de servir ses buts et ses intérêts.

En termes institutionnels, Fishkin est bien conscient de ce qu'il déplace la scène où s'élaborent les politiques et où se prennent les décisions. Le sondage d'opinion délibératif dresse le portrait de l'opinion du public informé à I'intention des représentants élus et de l'électorat entier. Pourtant, en termes normatifs, le portrait de l'opinion informée que brosse un sondage d'opinion délibératif représente déjà la volonté démocratique : «L'enquête résultante offre une représentation des jugements réfléchis du public - les points de vue auxquels arriverait le pays entier s'il faisait de même l'expérience de se comporter davantage comme des citoyens idéaux s'immergeant dans les problèmes débattus durant une longue période ${ }^{56} \mathrm{D}$ ans la mesure où la politique est devenue le "gouvernement par l'opinion publique» ${ }^{57}$, les sondages d'opinion délibératifs confèrent une légitimité démocratique aux décisions. II vaut la peine de noter, cependant, les nombreuses formes de rhétorique, de persuasion et et de formation discursive de la volonté que laisse de côté le sondage d'opinion délibératif, celui-ci se concluant en solitaire par des réponses à un questionnaire.

\section{Les sondages d'opinion délibératifs vus à travers la lorgnette d'une ontologie sociale relationnelle}

Dans les pages qui précèdent, nous avons vu comment certains traits clés du cas britannique - les liens de groupe, la dynamique de pouvoir, la dynamique de la conversation, le rôle de l'élite dans tout le processus - sont minimisés ou représentés de manière inadéquate en raison de l'ontologie sociale implicite de Fishkin. M ais le modèle de Fishkin peut-il accueillir une ontologie sociale relationnelle? En quoi faudrait-il le modifier pour qu'il y parvienne?

Fishkin n'affirme pas que les sondages d'opinions délibératifs constituent la référence obligée pour toutes les institutions démocratiques : en effet, il est clair que ce type particulier d'exercice vise principalement à recueillir des renseignements parmi une gamme élargie de corps représentatifs et décisionnels. Pourtant, même si l'on tient compte de la place modeste impartie aux sondages d'opinion délibératifs dans toute la gamme des institutions démocratiques possibles, négliger la dynamique des appartenances de groupe et les conceptions que les groupes ont d'eux-mêmes entame la légitimité normative des sondages délibératifs en tant que représentations de la gamme des intérêts et des points de vue dans la société civile.

Simultanément, les limites mêmes de la description que donne Fishkin de la délibération sont instructives, car elles fournissent un contexte concret

56. Fishkin, 1995, p. 162.

57. Fishkin voit en cette formule, due à J ames Bryce au XIX ${ }^{\mathrm{e}}$ siècle, la préfiguration de ce que la démocratie américaine allait devenir et signale combien la formulation de Bryce a eu de l'importance lorsque G eorge Gallup a cherché à justifier ses innovations en matière de sondages d'opinions. Voir Fishkin, 1995, p. 71-77. 


\section{4 - Philosophiques / Automne 2002}

permettant de voir en quoi une ontologie sociale façonne la constitution des institutions; nous pouvons alors nous pencher sur ce que cela pourrait signifier que de passer en revue les diverses phases des sondages d'opinion délibératifs à la lumière d'une meilleure appréciation de la dynamique des appartenances de groupe, de la formation de l'opinion et de la représentation. Explorons donc quelques modifications aux sondages d'opinion délibératifs susceptibles de leur faire mieux relever les défis de communication que posent les différences entre groupes. Les réflexions suivantes - là encore liées à l'exemple du sondage délibératif sur le système de justice pénale du Royaume-Uni - se veulent de simples suggestions : mon propos n'est pas de donner aux sondages délibératifs une nouvelle structure inattaquable, mais plutôt de montrer quel genre de préoccupations et de mécanismes occupent l'avant-scène quand on adopte une ontologie sociale relationnelle plutôt qu'individualiste.

\section{La sélection des participants}

J'ai suggéré que la sélection de Fishkin d'une coupe transversale de la population en vue de participer aux sondages délibératifs reflète son individualisme : une ontologie sociale davantage basée sur les groupes inciterait à porter une attention accrue à ceux qui sont généralement marginalisés lors des délibérations et ceux que le contexte particulier d'une politique affecte au premier chef.

O utre la défense pure et simple de son ontologie sociale individualiste, deux ripostes à cette suggestion s'offrent à Fishkin. Pour commencer, il peut défendre la sélection aléatoire des participants sur la base de considérations pragmatiques liées à la légitimité. Tant les critiques sociales collectivistes que relationnelles critiquent l'hégémonie de l'individualisme dans la politique contemporaine, y compris dans les conceptions de l'égalité et de l'équité en matière de représentation ${ }^{58}$. $M$ ais cela suggérerait que dans les contextes contemporains, l'approche de Fishkin de la sélection des participants cerne les normes de justice dominantes, ce qui confère de la crédibilité à l'issue des sondages délibératifs. On pourrait faire valoir un argument du même ordre sur la façon dont les exercices sont construits comme des modes sophistiqués de sondage de l'opinion, et ce dans un contexte où - comme le signale Fishkin - la politique se fait le plus souvent à coup de plébiscites. De plus, Fishkin pourrait faire valoir que les autres modes de sélection des participants que suggèrent mes critiques sont eux-mêmes problématiques, puisqu'ils nous obligent à porter des jugements de fond tant sur la façon dont les rapports de pouvoir décident de l'influence relative des groupes que sur les groupes affectés au premier chef par telle ou telle politique. Considérant que de tels jugements sont fort complexes et prêtent à discussion, I'approche de Fishkin a au moins l'avantage de la simplicité.

58. Voir Williams, 1998, chap. 2. 
M ême face à ces ripostes, je pense qu'il y a des arguments puissants en faveur de critères qui soient plus sensibles au contexte, de manière à porter une attention particulière à ceux que touche au premier chef un problème donné et à ceux qui sont en général marginalisés lors des délibérations menées au sein du courant majoritaire sur cette question. Cette tâche s'impose en raison de la reconnaissance, au sein d'une ontologie sociale relationnelle, de la dynamique de pouvoir entre groupes, laquelle peut prendre la forme de I'oppression et de la marginalisation ${ }^{59}$. Introduire les notions de différences de pouvoir et d'influence - de marginalisation et d'oppression, de perspectives traitées comme la norme et de perspectives réduites au silence, négligées ou mal comprises - modifie notre opinion sur l'identité et le nombre de ceux dont la présence s'impose le plus dans un sondage d'opinion délibératif. À cet égard, trois préoccupations particulières émergent quant au genre de critères susceptibles de nous guider dans le choix des participants invités à délibérer selon une approche relationnelle. Pour commencer par le point plus évident, il est certain que des perspectives propres à un groupe sont susceptibles d'être négligées lors des séances d'information et des délibérations à moins que des représentants de ce groupe ne soient eux-mêmes présents lors des discussions et de l'élaboration du processus. Ensuite, certaines perspectives propres à un groupe sont susceptibles d'être ignorées à moins que des membres de ce groupe ne soient présents en nombre suffisant lors des discussions; or, la structure des sondages d'opinion délibératifs prévoit que plusieurs centaines de participants sont scindés aléatoirement en groupes d'une vingtaine de personnes. Enfin, dans le cadre d'une ontologie sociale relationelle, il est important de tenir compte du pluralisme interne des groupes sociaux et de la façon fluide et changeante dont les identités et les intérêts se négocient entre membres des groupes sociaux. Cela suggère aussi que dans la mesure où la perspective du groupe est particulièrement saillante pour la question débattue, il faudrait que certains de ses membres soient présents en nombre significatif.

La délibération inclusive peut façonner le processus dès les premières étapes : il faudrait que les organisateurs rassemblent une équipe aussi diverse que possible pour mettre en branle l'élaboration du processus et favoriser une réflexion soutenue sur les voix qu'il est nécessaire d'entendre de même que sur les obstacles potentiels à l'inclusion de celles-ci. Ceci nous amène à nous pencher sur les problèmes particuliers à l'étude. Lors de l'élaboration d'un sondage délibératif sur le système de justice pénale, par exemple, il serait nécessaire que les organisateurs se demandent quels groupes ont joué un rôle central dans la définition des idées reçues et l'action politique en matière de justice pénale :

59. II y a une vaste littérature sur les appartenances de groupe et la représentation. Ce serait sortir du cadre de cet article que de la passer en revue ici. Voir par exemple Phillips, Anne, The Politics of Presence, Oxford, Clarendon Press, 1995; Williams, 1998; Young, Justice and the Politics of D ifference. 


\section{6 · Philosophiques / Automne 2002}

Q uelle est la gamme des points de vue émergeant du courant dominant? Q uelles sont les perspectives marginalisées et exclues? Cette dernière catégorie peut comprendre les communautés d'immigrants et les personnes de couleur, les détenus et groupes de défense des droits des détenus et les victimes d'actes criminels. Ces groupes peuvent aussi - en raison de leur marginalisation - être fondés à se dire particulièrement touchés par les décisions en matière pénale. $0 \mathrm{n}$ le voit, le processus est difficile et prête le flanc à la discussion dès cette étape. Et pourtant, le fait même qu'il prête à discussion indique bien quelles sont les limites des approches plus faciles de l'élaboration du processus (parce que moins démocratiques et moins réfléchies).

Les organisateurs feraient face à des décisions difficiles au moment de sélectionner les participants. D'une part, l'exercice cherche à fournir un modèle de délibération reflétant la diversité sociale : cela suggère une approche de la sélection des participants qui soit transversale, conformément à ce qui s'est produit dans la plupart des sondages délibératifs à ce jour. D'autre part, certains groupes et perspectives sont particulièrement touchés par le système de justice pénale et risquent tout particulièrement d'être peu ou mal entendus. II faut non seulement que des représentants de ces groupes soient présents, mais qu'ils le soient en nombre suffisant pour faire bloc, résister aux récits dominants et se faire entendre lors des délibérations. Lors du sondage de 1994, la sélection aléatoire a fait en sorte que deux anciens condamnés se sont retrouvés parmi les trois cents participants à la délibération. Une réflexion de fond sur les problèmes d'appartenances de groupe, de points de vue et de pouvoir aurait vraisemblablement dicté un «suréchantillonnage» de ceux qui ont ainsi une expérience directe du système de justice pénale. Un tel «suréchantillonnage» peut être également de mise quand certains groupes ethniques sont surreprésentés dans la population carcérale et sous-représentés au sein des instances judiciaires, ce qui leur rend manifestement plus vulnérables face à un système dominé par les blancs et dans bien des cas raciste.

II vaut la peine de noter que lors de l'élaboration d'au moins un sondage d'opinion délibératif, on a reconnu qu'il fallait aller au-delà des critères de représentativité statistique. Un organisme appelé I ssues D eliberation Australia a organisé un sondage d'opinion délibératif en 2001 sur la réconciliation entre $A$ ustraliens aborigènes et non aborigènes. En reconnaissance du caractère central des perspectives aborigènes sur cette question et de la diversité interne de la communauté, il y a eu «suréchantillonnage» des participants aborigènes de manière à ce qu'ils forment environ 12 pour cent des participants au sondage, et non pas 2 pour cent, ainsi qu'on aurait dû s'y attendre si l'échantillonnage s'était fait de manière aléatoir ${ }^{60}$. O n pourrait de même construire des arguments en faveur du «suréchantillonnage» de groupes d'intérêts touchés plus que les autres par telle ou telle question politique et courant plus que les autres le risque de voir leurs perspectives ignorées.

60. Voir http ://www.i-d-a.com.au/recon_report.htm. 
Comme on l'a déjà noté, prévoir à dessein l'inclusion de tel ou tel groupe rend complexes et discutables toutes les étapes de l'élaboration des sondages d'opinion délibératifs, alors qu'un échantillonnage aléatoire s'accorde bien mieux avec les présupposés de bien des démocraties contemporaines. Pour qui a adopté une ontologie sociale individualiste, la représentation dans le cadre d'un sondage d'opinion délibératif est affaire de représentativité statistique de l'échantillon : il n'est pas nécessaire de faire face ni à la complexité, ni à la surpolitisation des questions d'appartenances à des groupes sociaux, de marginalisation et d'oppression. $M$ ais d'un point de vue relationnel, les modes individualistes de sélection des participants échouent à satisfaire aux normes d'égalité ou de légitimité démocratiques : I'élaboration des institutions délibératives doit explicitement tenir compte des rapports de pouvoirs entre groupes pour que le dialogue inclue ceux qui doivent l'être. C'est dire que le modèle individualiste même apparaît comme un geste politique, quoiqu'il concorde mieux avec les conceptions et les intérêts des groupes sociaux dominants et leur convienne davantage.

Si introduire des critères basés sur l'oppression et la marginalisation dans les processus de sélection des participants a la vertu de mettre en lumière le caractère discutable des décisions sur l'identité des groupes à inclure, cela ne simplifie en rien ces décisions. Q ui est le plus touché par les décisions en matière pénale? Q uels ont été les groupes injustement marginalisés lors de la conception et la justification du système existant ou qui doivent être nommément inclus lors des discussions sur sa réforme? Les N oirs sont grandement surreprésentés dans les prisons britanniques; est-ce là un indice de marginalisation et d'oppression suggérant qu'il faille les suréchantillonner lors du sondage d'opinion délibératif sur cette question? Q u'en est-il de la prédominance des hommes par rapport aux femmes dans le système carcéral ? C ela indiquet-il là encore une dynamique de marginalisation et d'oppression justifiant de manière analogue un suréchantillonnage? N ous sommes nécessairement renvoyés à des analyses conceptuelles fort discutables sur le pouvoir et l'oppression et sur la façon dont celles-ci valent pour des sociétés et des contextes politiques donnés. Ce qui complique encore les choses en amont, c'est que même si on avance des assertions défendables sur les groupes considérés comme marginalisés et opprimés pour les fins de représentation d'un sondage donné, la question des frontières entre les groupes et de l'identité de ceux qui peuvent adéquatement représenter leur perspective peut encore donner lieu à un intense conflit.

Je ne puis offrir de solution élégante à ces questions piégées. J e souhaite plutôt souligner que passer d'une ontologie sociale individualiste à une ontologie relationnelle les fait ressurgir de manière insistante. Et dans la mesure où les approches relationnelles, à juste titre, pointent du doigt la nature politique de l'individualisme lui-même, on gagne sur les plans épistémique et normatif à ce que la contestation et la complexité de la représentation apparaissent au grand jour. Comme jel'ai noté plus haut, cependant, il se peut qu'il n'existe aucun moyen d'éviter que les sondages d'opinion délibératifs n'y perdent en 


\section{8 - Philosophiques / Automne 2002}

légitimité, si tant est que l'individualisme reflète davantage les conceptions populaires au sein d'une société démocratique donnée. Les défenseurs d'une approche relationnelle ne devraient pas glisser allègrement sur ce qu' elle coûte en complexité et en légitimité, pas plus que sur les difficultés à faire valoir ses analyses conceptuelles du pouvoir et de l'oppression ou les prétentions d'un groupe particulier s'estimant marginalisé et opprimé. Dans une perspective relationnelle, toutefois, il peut y avoir des retombées positives à ouvrir ainsi des discussions sur des questions épineuses de pouvoir, d'oppression et d'inclusion à propos des sondages d'opinion délibératifs. Pour commencer, les sondages d'opinion délibératifs peuvent devenir plus justes et être mieux en mesure de satisfaire aux normes d'inclusion démocratiques. Ensuite, quoi qu'il puisse nous en coûter en légitimité de renoncer à la représentativité statistique, ce ne sont pas toujours les mêmes qui auront à en faire les frais : les groupes habitués à être marginalisés lors des délibérations au sein du courant dominant peuvent trouver leur compte à prendre part aux sondages d'opinion délibératifs s'ils y sont représentés en nombre significatif. Enfin, au plan démocratique, on peut également gagner à ce que les délicates questions de pouvoir et d'inclusion soient débattues, en particulier quand le débat est à la fois inclusif et récursif, et donc quand il n'est pas l'apanage des membres de l'élite chargés d'élaborer les sondages d'opinions délibératifs, mais forme le terrain d'une contestation délibérative plus vaste ${ }^{61}$.

\section{En attendant la délibération}

En dévoilant l'individualisme des sondages d'opinion délibératifs tels que Fishkin les conçoit actuellement, j'ai noté deux problèmes concernant l'intervalle entre le moment où les participants sont incités à entrer dans le processus et le stade délibératif. L'un des problèmes, c'est que la trousse d'information étant élaborée par l'élite, cela risque de perpétuer la marginalisation de certaines perspectives. Fishkin et Luskin répliquent à cette critique en ces termes:

N os répondants ne sont pas dans une «situation idéale de parole» à la $\mathrm{H}$ abermas où ils auraient un temps illimité pour se livrer à la discussion. Ils se préparent raisonnablement bien à un long week-end. $N$ otre but est de leur fournir une meilleure information, pas une information parfaite. N ous croyons qu'on peut préparer une trousse d'information qui soit relativement exacte, équilibrée et accessible aux citoyens ordinaires ${ }^{62}$.

Si cet argument peut justifier la clause sur les limites nécessaires de l'information, il ne justifie pas qu'on retienne une gamme particulière de rensei-

61. Cette insistance pour que le processus de délibération lui-même soit ouvert à des remises en question discursives fait écho tant à l'analyse critique du travail de Carol Gilligan par $\mathrm{H}$ eyes (voir H eyes, Cressida, Line D rawings. D efining Women through Feminist Practices, Ithaca, Cornell University Press, chap. 5, 2000) qu'à la description que donne $\mathrm{H}$ abermas des idéaux de la discussion. Voir H abermas, 1990.

62. Fishkin et Luskin, 1996, p. 48. 
gnements ni ne plaide contre l'inclusion d'un large spectre de renseignements dans la trousse d'information. Aussi peut-on concéder qu'aucun processus n'est parfait, mais réitérer néanmoins l'importance d'un processus démocratique inclusif et récursif lors de l'élaboration d'un sondage d'opinion délibératif, y compris au moment où est constituée la trousse d'information.

Le second problème de Fishkin qui est inhérent à la conception individualiste de l'intervalle entre l'invitation à participer et la délibération a trait à la représentativité des participants. Selon une ontologie sociale relationnelle, il n'y a pas d'intérêts ou de perspectives statiques à représenter (au nom des individus ou des groupes). Ainsi, la forme de l'interaction entre les participants et leurs communautés constitutives est centrale, y compris durant les semaines précédant la délibération. Ici aussi, Fishkin et Luskin ont une réponse toute prête : le délai entre le recrutement initial en vue du sondage d'opinion délibératif et l'événement lui-même donne aux participants le loisir de colliger des renseignements de leur propre chef. Discutant de plans en vue d'un sondage d'opinion délibératif sur des questions d'intérêt national à Austin en 1996, Fishkin et Luskin écrivent :

[... ] en un sens important, l'intervention s'étend au-delà - et se fait de manière plus naturelle - du week-end à Austin. Les participants sauront dès leur recrutement qu'ils assisteront à un événement télédiffusé dans toute la nation pour discuter de politique. Durant les quelque quatre à dix semaines précédant le [congrès sur des questions d'ordre national], ils liront davantage sur la politique, écouteront les bulletins plus attentivement et parleront davantage de politique avec leur famille et leurs amis ${ }^{63}$.

Les participants auront accès à une information plus diversifiée que celle comprise dans la trousse d'information. Et dans la mesure où leur capacité à représenter ceux qui occupent la même place dans la société dépend de leur implication auprès de ceux qui occupent une place similaire, ils peuvent y parvenir, en attendant l'événement, en choisissant pour interlocuteurs ceux qui hantent leurs cercles sociaux et politiques habituels.

Cette dernière réponse est à bien des égards intéressante, en particulier si on la combine à une ouverture du processus d'élaboration du débat de façon à y inclure des voix diverses. On peut également se demander jusqu'à quel point des processus liés à la sélection des participants et à la conception de la trousse d'information qui soient plus politisés peuvent modifier la conception que les participants se font de leur tâche. La nature représentationnelle de leur participation serait plus explicite, et cela peut en soi les pousser à prendre l'initiative de s'impliquer avec autrui en prévision de la délibération formelle et pousser les membres de leurs groupes constitutifs - à titre individuel ou de manière plus organisée - à prendre l'initiative de se pencher sur les perspectives du groupe et sa représentation.

63. I bid. 


\section{0 - Philosophiques / Automne 2002}

\section{La localisation de la délibération}

Reconnaître l'importance des appartenances de groupe et des rapports de pouvoir entre groupes a un impact sur la façon dont nous relions à leur communauté les individus appelés à délibérer. J'ai suggéré plus tôt qu'isoler les participants de leur communauté constitue un problème particulier pour la mise en œuvre des sondages délibératifs. O n pourrait modifier les suggestions de Fishkin en conséquence.

La mise à l'écart des identités et affinités propres à un groupe ne survient pas seulement quand on isole les participants de leur communauté habituelle le temps de la délibération, mais aussi lors de l'élaboration des procédures de délibération elles-mêmes. Les participants se réunissent pour poser des questions aux experts puis se dispersent dans des groupes de discussion divisés de manière aléatoire afin de réfléchir ensemble à l'information qu'ils ont recueillie. Les participants aux groupes de discussion en viennent à « se connaître assez bien pour délibérer ensemble» 64 . Ils interagissent de manière intense plusieurs jours durant, partageant leurs repas et bâtissant un lien de confiance mutuelle à travers la discussion et le dialogue.

$M$ ais en quel sens cette réunion d'individus recrée-t-elle la délibération face à face telle qu'elle se déroulait dans la polis antique ou ailleurs? D ans ses formes les moins artificielles, la délibération face à face n'est ni individualiste (opposant des agents discrets partageant de l'information), ni uniformément solidaire (les rapports de confiance et de compréhension entre participants étant symétriques). La délibération face à face comporte des sous-groupes, des sous-solidarités et des enclaves, lesquels jouent tous un rôle clé. L'un des rôles importants joués par les sous-groupes est de formuler des points de vue en porte-à-faux avec les perspectives dominantes. Cela est particulièrement important là où les groupes sont traditionnellement exclus ou marginalisés, de sorte que leurs opinions ont moins de chances d'être thématisées ou prises au sérieux lors des délibérations et que leur style de communication a moins de chances de passer la rampe ${ }^{65}$. Tant que les membres de tels groupes sont maintenus séparés dans le cadre de délibérations hétérogènes, il peut être difficile pour eux de formuler leurs positions, de reconnaître à quel titre ils sont marginalisés

64. Fishkin, 1991, p. 92.

65. Young fait valoir, par exemple, que la politique américaine privilégie les discours assurés et agressifs au détriment de ceux qui sont plus timides et conciliants; les discours généraux formels au détriment des discours hésitants et tortueux; les discours prononcés sans passion au détriment de ceux chargés d'émotion et ponctués de gestes éloquents. Toutes ces normes sur les discours faisant autorité privilégient les groupes dominants tout en dévaluant les raisons fournies par les autres. Voir Young, Iris M arion, 2000, chap. 2 et 3; voir aussi Fraser, $\mathrm{N}$ ancy, « R ethinking the Public Sphere. A Contribution to the Critique of Actually Existing Democracy », dans Robins, B., dir., The Phantom of Public Sphere, M inneapolis, University Press, 1993; Fraser, N ancy, «Toward a Discourse Ethic of Solidarity», Praxis I nternational, vol. 5, n 4, 1986; M ansbridge, Jane, «Feminism and Democratic Community», dans Chapman, J. W. et Shapiro, I., dir., N omos XXX V. D emocratic Community, N ew York, N ew York University Press, 1993, p. 339-395. 
ou de développer des stratégies afin de contrer cette marginalisation ${ }^{66}$. En outre, il peut y avoir de puissants incitatifs pour les membres des groupes marginalisés à minimiser leur différence, histoire de s'intégrer.

Ces dynamiques plaident en faveur de l'instauration de mécanismes permettant à des groupes particuliers de former un bloc. On pourrait désigner d'avance ces groupes à la lumière d'une vaste analyse des rapports de pouvoir et d'exclusion. De tels groupes ou «enclaves» permettraient de développer, raffiner et reformuler des opinions en porte-à-faux avec les perspectives dominantes au cours des délibérations, au point de faire éventuellement leur apparition lors de discussions plus hétérogènes ${ }^{67}$.

Une objection possible contre de tels blocs est que cela créerait des factions lors des délibérations. Certaines positions émergeraient non pas de manière organique, au fil des discussions et des débats, mais parce qu'elles ont été énoncées par le «bloc noir » ou le «bloc des ex-détenus». Les positions se consolideraient autour de catégories identitaires plutôt que de se former plus naturellement autour des opinions des individus sur les questions à l'étude ${ }^{68}$. $M$ ais cette objection ne tient peut-être pas suffisamment compte du poids des inégal ités telles qu'elles se sont sédimentées dans les attitudes des participants et la dynamique de leur interaction. Les affinités et les identités des participants liées au groupe ne disparaissent pas quand elles ne sont pas explicitement thématisées. Elles agissent plutôt sur la dynamique des conversations et sur les accords qui en émergent.

\section{L'éducation des participants}

Lorsque j'ai discuté des propositions de Fishkin, je me suis dit préoccupé de ce qu'il confie d'un bout à l'autre l'exercice de délibération à des membres de I'élite et me suis inquiété de la sélection et du rôle des experts appelés à se prononcer lors du processus. Dans la mesure où on prend au sérieux les limites épistémiques qui sont les nôtres en tant que membres de groupes sociaux et

66. Le fait que les experts et les témoins invités représentent inévitablement des perspectives liées à des groupes peut constituer une ressource positive quand on se penche sur les questions d'identité de groupe et de délibération discutées ci-dessus. Ces invités peuvent être sélectionnés pour formuler des perspectives propres à un groupe qui seraient sinon négligées ou marginalisées au cours des délibérations.

67. Sur l'importance des «enclaves» pour les processus démocratiques, voir Jane M ansbridge, Jane, «Using Power/Fighting Power », Constellations, vol. 1, $n^{0} 1$ 1, 1993, p. 53-73.

68 . Il semble vraisemblable que cette polarisation pourrait être exacerbée par la période de contact limitée entre participants : c'est dans un contexte où peut s'épanouir une culture d'ouverture capable d'examiner ses propres préjugés que les mécanismes de délibération s'appuyant sur les groupes ont le plus de chances de réussir. Les organisations féministes et de gays et lesbiennes offrent des cas exemplaires d'usage productif (et parfois quelque peu polarisant) des blocs afin de surmonter des inégalités et des préjugés tenaces. Voir Sirianni, Carmen, «L earning Pluralism. Deocracy and Diversity in Feminist O rganizations», dans Chapman, J. W. et Shapiro, I., dir., N omos X XXV. D emocratic Community, N ew York, N ew York University Press, 1993, p. 283-312. 
le rôle que joue le pouvoir dans l'obscurcissement de ces limites aux yeux de certains, on a de bonnes raisons de thématiser les différences entre groupes avant que le sommet ne commence effectivement et de faire appel aux membres des divers groupes au moment de négocier les questions relatives aux experts, à la connaissance et à l'élaboration du processus. Une telle démocratisation réfléchie de l'élaboration des sondages délibératifs jouerait selon toute vraisemblance sur le choix des voix qui se feraient entendre durant les séances plénières. Lors du sondage délibératif sur le système de justice pénale au Royaume-Uni, par exemple, les participants ont eu la chance d'interroger « des universitaires, des hauts gradés de la police, des avocats, des spécialistes de la prévention du crime et de la délinquance juvénile ainsi qu'un individu reconnu coupable d'un crime pour mettre à l'épreuve les questions ayant surgi lors des discussions antérieures ${ }^{69}$. La gamme et l'identité des voix faisant autorité peuvent être bien différentes selon qu'on élabore de telle ou telle manière le processus de démocratisation et de politisation et qu'on adopte telle ou telle conception de ce que c'est qu'être un expert en la matière.

La structuration de la conversation

J'ai déjà rel evé l'une des façons dont une ontologie sociale relationnelle suggère des modifications dans la structure de délibération : plutôt que de traiter les participants comme de simples individus, les organisateurs doivent ménager une place aux asymétries de pouvoir, de solidarité et de connaissance fondées sur les groupes en prévoyant la formation de blocs et d'autres mécanismes. Les défis que posent de telles asymétries pour la communication par-delà les différences entre groupes suggèrent de nouvelles modifications d'ordre institutionnel : les animateurs et les modérateurs jouent ici un rôle clé, comme Fishkin pourrait bien le reconnaître. $N$ ous disposons de nombreux outils au plan théorique et pratique pour faciliter la discussion dans un contexte où les différences sociales sont profondes et où le partage des pouvoirs est asymétrique. S'ils sont sensibles aux différences de pouvoir entre participants et aux divers styles de communication, ces animateurs peuvent jouer un rôle important de médiation entre groupes aux pouvoirs inégaux, servant par là les normes d'égalité ${ }^{70}$. Ces mesures et d'autres encore serviraient à thématiser les questions de différences entre groupes, de répartition asymétrique du pouvoir, de normativité et d'autorité pour les participants. La délibération collective a alors des chances d'être plus décoiffante, et elle suppose qu'on s'attache tant au processus qu'au contenu.

69. Fishkin, 1994, p. 8.

70. Les contributions théoriques et pratiques sur la résolution de débats interculturels sont particulièrement pertinentes ici. Pour une étude utile de la littérature, voir LeBaron, M ichelle, Conflict and Culture. A Literature R eview and Bibliography, Victoria, University of Victoria Institute for Dispute R esolution, 1992. 


\section{La conclusion de l'exercice}

Des mécanismes de délibération peuvent être développés pour relever non seulement les défis que pose la communication par-delà les différences, mais aussi ceux relatifs à la conclusion d'un accord. Les mécanismes d'accord portent entre autres sur les exigences que doivent remplir les consensus, les droits de veto, les votes séquentiels, les votes à main levée et les surmajorités. 0 n trouvera d'autres modèles pertinents dans l'abondante littérature sur les modèles non standards de résolution de différends ${ }^{71}$. Le sondage d'opinion délibératif de Fishkin ne prévoit aucune place pour de tels mécanismes, celuici se concluant avec l'administration d'un questionnaire que les participants remplissent chacun de leur côté.

Comme nous en avons fait état plus haut, il peut y avoir des questions pragmatiques de légitimité et d'influence qui justifient qu'on donne à l'exercice de délibération la forme d'un sondage sur l'opinion informée des individus. II vaut toutefois la peine de noter comment opter pour un sondage dispense les participants de devoir convaincre autrui avant de prendre leur décision ou d'assumer publiquement la responsabilité de leur propre position : il n'y a pas de moment où les décisions sont prises collectivement et les opinions sont relevées individuellement. Cela altère profondément la dynamique de la délibération, car il appartient alors à chaque individu de décider à quel point il soumettra son opinion aux critiques d'autrui et dispense chacun de l'obligation de mettre de l'eau dans son vin afin d'arriver à une solution commune. II s'agit d'une délibération sans négociation, ce qui ferme par là la porte à bien des formes de dial ogue et de formation subjective de la volonté typiques des autres exercices démocratiques.

La conception que se fait Fishkin de la démocratie suggère que les décisions à prendre devraient être guidées par la «voix du peuple», qu'il décrit comme l'agrégat des opinions individuelles informées. M ais cette conception de la démocratie ne prévoit pas de moment où se prennent les décisions. C'est dire que ma critique met au jour une tension profonde dans le modèle de Fishkin. Le sondage et la délibération sont des activités bien différentes, la première culminant en une indication de l'opinion individuelle détachée de tout contexte, la seconde entraînant la négociation d'une perspective partagée en tout ou en partie. Fishkin néglige par là la disjonction importante qu'il y a entre le sondage délibératif et les exercices de prise de décision face à face. II veut soutenir que les sondages délibératifs comme celui sur le système de justice pénale britannique « ont démontré la viabilité d'une forme de sondage de l'opinion différente et, en un sens, d'une forme de démocratie différente» 72 . En effet, il affirme que les sondages d'opinion délibératifs ont pour ancêtre une

[... ] forme de représentation qui gît cachée dans la poussière de l'histoire. Elle a été employée par les commissions législatives, les jurys de citoyens et le conseil de

71. LeBaron, 1992.

72. Fishkin, 1995, p. 168. 


\section{4 - Philosophiques / Automne 2002}

I'A thènes antique (ce dernier jouant le rôle crucial de déterminer l'ordre du jour des réunions de l'assemblée des citoyens). Cette autre méthode était la sélection par tirage au sort ou par échantillonnage aléatoire. En un sens, I'utilisation d'échantillons aléatoires a été ressuscitée par le sondage d'opinion. A près tout, qu'est-ce qu'un sondage d'opinion, au fond, sinon une loterie? M ais sous sa forme grecque antique, et sous la forme employée lors du sondage délibératif, on relève non pas l'opinion de citoyens isolés, mais celle de citoyens se réunissant pour délibérer de problèmes communs. Ces sondages représentent les jugements réfléchis de l'assemblée, pas les réactions lancées en l'air par des citoyens isolés. Les institutions qui parlent au nom du peuple doivent être à la fois représentatives et délibératives. L'innovation de la G rèce antique a été de réunir aléatoirement un échantillon de cityens discutant ensemble, réalisant de cette façon les deux valeurs. Et c'est cette forme que je propose d'adapter à l'âge de la télévision ${ }^{73}$.

L'innovation de Fishkin est en effet représentative en un sens important, mais par ses composantes délibératives, elle s'écarte significativement du modèle athénien et d'autres expériences démocratiques en ce qu'elle ne comporte pas de moment où sont prises les décisions.

Dès lors que l'élaboration du sondage délibératif cesse d'être la prérogative d'experts comme Fishkin et qu'elle se démocratise en devenant plus inclusive, le mode de conclusion de l'exercice - comme toutes les autres facettes du processus - est sujet à une remise en question. M ême à supposer que les mécanismes de sondages soient retenus, ce serait à la lumière de discussions sur le statut de leurs résultats. II faudrait en effet se demander en quel sens ils représentent ou non ce à quoi ressemblerait I'opinion publique «si les répondants étaient motivés à surmonter les incitatifs les poussant à l'ignorance rationnelle pour se comporter un peu plus comme des citoyens idéaux $\gg^{74}$.

En quoi les résultats d'un tel sondage d'opinion délibératif différeraientils de ceux d'un sondage s'appuyant (à l'instar du sondage de justice pénale du Royaume-Uni) sur des présupposés individualistes? On ne peut qu'avancer des conjectures sur la façon dont les connaissances et les préférences politiques des participants seraient modifiées si l'on changeait ainsi le processus: le changement le plus évident concernerait l'autorité dont le sondage d'opinion délibératif pourrait se réclamer comme représentation de l'opinion publique, de même que la place que de tels sondages pourraient prendre dans l'ensemble des délibérations et des contestations auxquelles donnent lieu la réforme de la justice pénale.

Q u'on se rappelle deux affirmations de Fishkin à la défense des sondages d'opinion délibératifs. La première porte sur la représentativité des participants : les sondages d'opinion délibératifs «se servent des techniques d'échantillonnage mises au point par la recherche sur l'opinion publique pour représenter et réunir une version de ce que nous sommes tous ${ }^{75}$. Cette affirmation est remise

73. Ibid., p. 169

74. Ackerman et Fishkin, 2000, p. 7.

75. Fishkin, 1991, p. 12. 
en question par les changements d'ordre méthodologique que je propose. $M$ ais dès lors qu'on nourrit des doutes sur l'ontologie sociale sous-tendant la théorie de Fishkin, elle est remise en question. Le «nous» démocratique n'est pas quelque chose de statique, ce n'est pas une collection d'individus (ni, d'ailleurs, de groupes) avec des identités et des intérêts préexistants. Le demos est plutôt une constellation changeante d'identités liées aux groupes se définissant à travers une perpétuelle contestation au sein des groupes et entre groupes. La prétention des sondages d'opinion délibératifs à présenter une « version de ce que nous sommes tous» n'a jamais été plausible. Les modifications que j'ai présentées ont simplement mis en lumière le caractère contingent et éminemment politique de toute prétention à représenter le peuple ou la volonté populaire. Ainsi, mon approche ouvre la porte à la contestation là où elle aurait toujours dû l'être, soit à propos de ce que «nous» sommes en tant que société démocratique et de ce que cela peut signifier pour un groupe ou pour une mesure politique de prétendre nous représenter.

Ensuite, Fishkin affirme que l'issue d'un sondage d'opinion est représentatif de l'électorat en un sens supérieur :

Un sondage d'opinion délibératif fournit le modèle de ce que l'électorat penserait si, par hypothèse, il pouvait s'immerger dans d'intenses processus de délibération. L'intérêt d'un sondage d'opinion délibératif, c'est qu'il est prescriptif, et non pas prédictif. II a une force de recommandation, nous disant ce que la masse entière du public penserait de certaines questions politiques ou de candidats si on lui donnait l'occasion de se livrer à une réflexion approfondie et un accès important à l'information ${ }^{76}$.

Là encore, l'effet des changements suggérés par une ontologie sociale relationnelle est de déstabiliser toute prétention en ce sens. Car dans la mesure où il y a contestation ouverte de la base de participation, de l'information fournie aux participants, de la forme de leur interaction et de la représentation de leurs opinions une fois transformées, on voit clairement à quel point la « réflexion » et l'« accès à l'information » sont éminemment manipulables. II n'y a rien de fatal ou d'objectif et d'incontestable dans la sélection des participants et la forme que doit prendre leur interaction. Ce sont là des questions politiques, étant donné la contestation à laquelle donnent lieu la façon de formuler et concevoir les sujets à débattre, l'identification des groupes touchés au premier chef, la dynamique de pouvoir parmi les groupes sociaux, etc.

Ainsi, en quoi les modifications suggérées par une ontologie sociale relationnelle changent-elles la place du sondage d'opinion délibératif sur le système de justice pénale si on le replace dans le paysage démocratique britannique? L'organisation et la mise en œuvre du sondage mettraient en branle toute une série de nouvelles délibérations et contestations complexes à l'issue imprévisible : on discuterait de la justification des modalités d'organisation du

76. I bid., p. 81. 


\section{6 - Philosophiques / Automne 2002}

sondage, du caractère adéquat de la représentation et du peuple, de l'équité des structures de délibération et de l'interprétation à donner des résultats. En d'autres mots, le sondage serait davantage que le résultat éventuel d'une réflexion et d'un débat entre universitaires : ce serait le ferment d'une société civile dynamique.

Les sondages d'opinion délibératifs de Fishkin sont une bonne chose. II nous en faut davantage. Les déficits au plan de la délibération qu'il identifie dans les démocraties libérales contemporaines sont réels et troublants, et toute contribution permettant aux citoyens de se parler longuement et de manière respectueuse par-delà les différences est bienvenue. Q ui plus est, les paramètres procéduraux prévus par Fishkin s'accordent davantage avec le courant libéral dominant que les révisions que j'ai suggérées. Car selon le courant dominant, l'équité signifie un traitement uniforme des individus (sans égard à la couleur, au sexe, à la différence), elle signifie qu'on traite les gens comme des individus. Toute tentative pour faire face aux inégalités et à la marginal isation tenaces de manière structurée a de bonnes chances de passer pour la promotion de «droits particuliers», de la «discrimination à rebours» ou de la « rectitude politique». C'est dire que les propositions de Fishkin ont de bien meilleures chances d'apparaître légitimes aux yeux du public, sans parler de leurs chances d'être financées, que tout ce que je suis susceptible de suggérer. Eu égard au sérieux du déficit au plan de la délibération qu'il a identifié, la viabilité des sondages d'opinion délibératifs aux yeux du courant dominant est manifestement une vertu.

$M$ ais les limites des sondages d'opinion délibératifs tels que les conçoit Fishkin apparaissent tout aussi clairement. Des délibérations équitables entre citoyens doivent prendre au sérieux non seulement les intérêts faciles à transposer en des termes compris de tous, mais aussi les expériences et les conceptions propres à un groupe qui peuvent être plus difficiles à communiquer. $\mathrm{Ce}$ défi à la communication par-delà la différence ressort d'autant étant donné la prévalence de l'injustice et des phénomènes de marginalisation dans la société civile. Prendre ce problème au sérieux, c'est faire place aux identités et aux allégeances lors de la délibération elle-même. II n'y a pas qu'une bonne ontologie sociale qui l'exige. La justice démocratique aussi $77,78$.

77. Tous mes remerciements à Cressida $\mathrm{H}$ eyes et $\mathrm{D}$ ominique Leydet, dont les commentaires m'ont été extrêmement utiles, et à Elizabeth Panasiuk, pour son aide au cours de la recherche.

78. Cet article a été traduit de l'anglais par Dominique Boucher. 